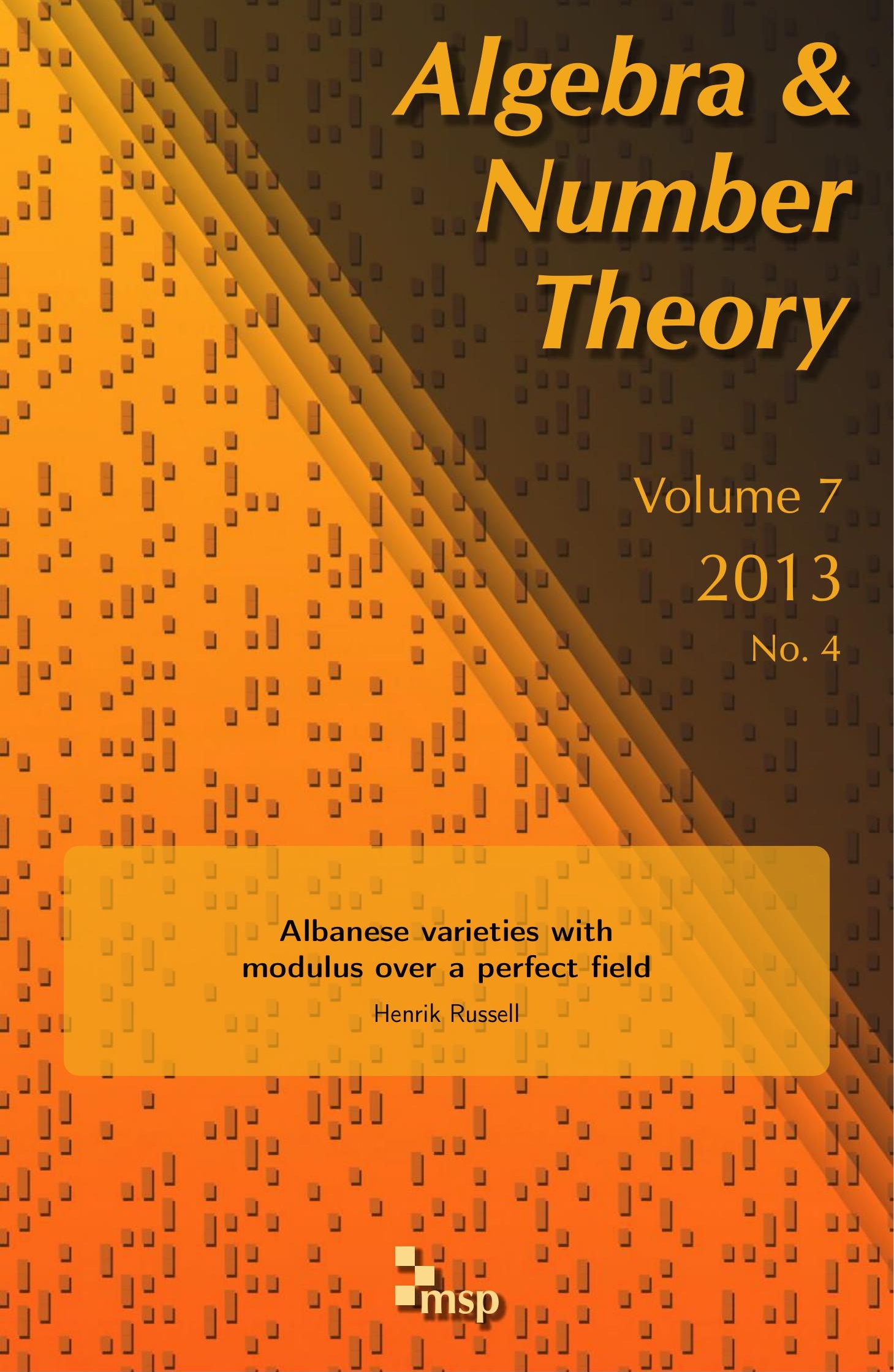




\title{
Albanese varieties with modulus over a perfect field
}

\author{
Henrik Russell
}

Let $X$ be a smooth proper variety over a perfect field $k$ of arbitrary characteristic. Let $D$ be an effective divisor on $X$ with multiplicity. We introduce an Albanese variety $\operatorname{Alb}(X, D)$ of $X$ of modulus $D$ as a higher-dimensional analogue of the generalized Jacobian of Rosenlicht and Serre with modulus for smooth proper curves. Basing on duality of 1-motives with unipotent part (which are introduced here), we obtain explicit and functorial descriptions of these generalized Albanese varieties and their dual functors.

We define a relative Chow group of zero cycles $\mathrm{CH}_{0}(X, D)$ of modulus $D$ and show that $\operatorname{Alb}(X, D)$ can be viewed as a universal quotient of $\mathrm{CH}_{0}(X, D)^{0}$.

As an application we can rephrase Lang's class field theory of function fields of varieties over finite fields in explicit terms.

\section{Introduction}

The generalized Jacobian variety with modulus of a smooth proper curve $X$ over a field is a well-established object in algebraic geometry and number theory and has shown to be of great benefit, for instance, for the theory of algebraic groups, ramification theory and class field theory. In this work we extend this notion from [Serre $1959, \mathrm{~V}]$ to the situation of a higher-dimensional smooth proper variety $X$ over a perfect field $k$. The basic idea of this construction comes from [Russell 2008] and is accomplished in [Kato and Russell 2012], both only for the case that $k$ is of characteristic 0 . Positive characteristic however requires distinct methods and turns out to be the difficult part of the story.

To a rational map $\varphi: X \rightarrow P$ from $X$ to a torsor $P$ under a commutative algebraic group $G$ we assign an effective divisor $\bmod (\varphi)$, the modulus of $\varphi$ (Definition 3.11). Our definition from [Kato and Russell 2010] coincides with the classical definition in the curve case as in [Serre 1959, III, Section 1]. For an effective divisor $D$ on $X$ the generalized Albanese variety $\operatorname{Alb}^{(1)}(X, D)$ of $X$ of modulus $D$ and the Albanese map $\operatorname{alb}_{X, D}^{(1)}: X \rightarrow \operatorname{Alb}^{(1)}(X, D)$ are defined by the following universal property:

MSC2010: primary 14L10; secondary 11G45, 14C15.

Keywords: Albanese with modulus, relative Chow group with modulus, geometric class field theory. 
For every torsor $P$ under a commutative algebraic group $G$ and every rational map $\varphi$ from $X$ to $P$ of modulus $\leq D$, there exists a unique homomorphism of torsors $h: \operatorname{Alb}^{(1)}(X, D) \rightarrow P$ such that $\varphi=h \circ \mathrm{alb}_{X, D}^{(1)}$. Every rational map to a torsor for a commutative algebraic group admits a modulus, and the effective divisors on $X$ form an inductive system. Then the projective $\operatorname{limit} \lim \operatorname{Alb}^{(1)}(X, D)$ over all effective divisors $D$ on $X$ yields a torsor for a proalgebraic group that satisfies the universal mapping property for all rational maps from $X$ to torsors for commutative algebraic groups.

The Albanese variety with modulus (Theorem 0.2 ) arises as a special case of a broader notion of generalized Albanese varieties defined by a universal mapping property for categories of rational maps from $X$ to torsors for commutative algebraic groups. As the construction of these universal objects is based on duality, a notion of duality for smooth connected commutative algebraic groups over a perfect field $k$ of arbitrary characteristic is required. For this purpose we introduce so called 1-motives with unipotent part (Definition 1.18), which generalize Deligne 1-motives [1971, Définition (10.1.2)] and Laumon 1-motives [1996, Définition (5.1.1)]. In this context, we obtain explicit and functorial descriptions of these generalized Albanese varieties and their dual functors (Theorem 0.1).

In a geometric way we define a relative Chow group of 0 -cycles $\mathrm{CH}_{0}(X, D)$ with respect to the modulus $D$ (Definition 3.27). Then we can realize $\operatorname{Alb}^{(1)}(X, D)$ as a universal quotient of $\mathrm{CH}_{0}(X, D)^{0}$, the subgroup of $\mathrm{CH}_{0}(X, D)$ of cycles of degree 0 (Theorem 0.3), in the case that the base field is algebraically closed. The relation of $\mathrm{CH}_{0}(X, D)$ to the K-theoretic idèle class groups from [Kato and Saito 1983] gives rise to some future study, but is beyond the scope of this paper. Using these idèle class groups, Önsiper [1989] proved the existence of generalized Albanese varieties for smooth proper surfaces in characteristic $p>0$.

Lang's class field theory of function fields of varieties over finite fields [Serre $1959, \mathrm{~V}]$ is written in terms of so called maximal maps, which appeared as a purely theoretical notion, apart from their existence very little seemed to be known about which. The Albanese map with modulus allows us to replace these black boxes by concrete objects (Theorem 0.4).

We present the main results by giving a summary of each section.

0.1. Leitfaden. Section 1 is devoted to the following generalization of 1-motives: A 1-motive with unipotent part (Definition 1.18) is roughly a homomorphism $[\mathscr{F} \rightarrow G]$ in the category of sheaves of abelian groups over a perfect field $k$ from a dual-algebraic commutative formal group $\mathscr{F}$ to an extension $G$ of an abelian variety $A$ by a commutative affine algebraic group $L$. Here a commutative formal group $\mathscr{F}$ is called dual-algebraic if its Cartier-dual $\mathscr{F}^{\vee}=\underline{\operatorname{Hom}}\left(\mathscr{F}_{\mathcal{F}}, \mathbb{G}_{\mathrm{m}}\right)$ is algebraic. 1 -motives with unipotent part admit duality (Definition 1.21). The dual of $[0 \rightarrow G]$ 
is given by $\left[L^{\vee} \rightarrow A^{\vee}\right]$, where $L^{\vee}=\underline{\operatorname{Hom}}\left(L, \mathbb{G}_{\mathrm{m}}\right)$ is the Cartier-dual of $L$ and $A^{\vee}=\operatorname{Pic}_{A}^{0}=\underline{\operatorname{Ext}}^{1}\left(A, \mathbb{G}_{\mathrm{m}}\right)$ is the dual abelian variety of $A$, and the homomorphism between them is the connecting homomorphism associated to $0 \rightarrow L \rightarrow G \rightarrow A \rightarrow 0$. In particular, every smooth connected commutative algebraic group over $k$ has a dual in this category. Moreover, these 1-motives may contain torsion.

Section 2: Let $X$ be a smooth proper variety over a perfect field $k$. In the framework of categories of rational maps from $X$ to torsors for commutative algebraic groups (Definition 2.8), we ask for the existence of universal objects (Definition 2.14) for such categories, that is, objects having the universal mapping property with respect to the category they belong to. Assume for the moment $k$ is an algebraically closed field. Then a torsor can be identified with the algebraic group acting on it. A necessary and sufficient condition for the existence of such universal objects is given in Theorem 2.16, as well as their explicit construction, using duality of 1-motives with unipotent part. (This was done in [Russell 2008] for $\operatorname{char}(k)=0$.) We pass to general perfect base field in Theorem 0.1 .

In particular we show the following: Let $\underline{\operatorname{Div}}_{X}$ be the sheaf of relative Cartier divisors, that is, the sheaf of abelian groups that assigns to any $k$-algebra $R$ the group $\underline{\operatorname{Div}}_{X}(R)$ of all Cartier divisors on $X \otimes_{k} R$ generated locally on Spec $R$ by effective divisors which are flat over $R$. Let $\underline{\mathrm{Pic}}_{X}$ be the Picard functor and $\operatorname{Pic}_{X}^{0, \text { red }}$ the Picard variety of $X$. Then let $\underline{\operatorname{Div}}_{X}^{0, \text { red }}$ be the inverse image of Pic ${ }_{X}^{0, \text { red }}$ under the class map cl : $\underline{\operatorname{Div}}_{X} \rightarrow \underline{\operatorname{Pic}}_{X}$. A rational map $\varphi: X \rightarrow G$, where $G$ is a smooth connected commutative algebraic group with affine part $L$, induces a natural transformation $\tau_{\varphi}: L^{\vee} \rightarrow \underline{\operatorname{Div}}_{X}^{\text {, red }}$ (Section 2.2.1). If $\mathscr{F}$ is a formal subgroup of $\underline{\operatorname{Div}}_{X}^{0, \text { red }}$, denote by $\operatorname{Mr} \mathscr{F}(X)$ the category of rational maps for which the image of this induced transformation lies in $\mathscr{F}$. If $k$ is an arbitrary perfect base field, we define $\operatorname{Mr}_{\mathscr{F}}(X)$ via base change to an algebraic closure $\bar{k}$ (Definition 2.13).

Theorem 0.1. Let $\mathscr{F}$ be a dual-algebraic formal $k$-subgroup of $\underline{\mathrm{Div}}_{X}^{0, \text { red }}$. The category $\operatorname{Mr}_{\mathscr{F}}(X)$ admits a universal object $\operatorname{alb}_{\mathscr{F}}^{(1)}: X \rightarrow \operatorname{Alb}_{\mathscr{F}}^{(1)}(X)$. Here $\operatorname{Alb}_{\mathscr{F}}^{(1)}(X)$ is a torsor for an algebraic group $\operatorname{Alb}_{\mathscr{F}}^{(0)}(X)$, which arises as an extension of the classical Albanese $\operatorname{Alb}(X)$ by the Cartier-dual of $\mathscr{F}$. The algebraic group $\operatorname{Alb}_{\mathscr{F}}^{(0)}(X)$ is dual to the 1-motive $\left[\mathscr{F}^{\rightarrow} \mathrm{Pic}_{X}^{0, \text { red }}\right]$, the homomorphism induced by the class map $\mathrm{cl}: \underline{\mathrm{Div}}_{X} \rightarrow \underline{\mathrm{Pic}}_{X}$.

Theorem 0.1 results from (the stronger) Theorem 2.16, which says that a category of rational maps to algebraic groups (over an algebraically closed field) admits a universal object if and only if it is of the shape $\operatorname{Mr\mathscr {F}}_{(}(X)$ for some dual-algebraic formal subgroup $\mathscr{F}_{F}$ of $\underline{\operatorname{Div}}_{X}^{0, \text { red }}$, and Galois descent (Theorem 2.21). The generalized Albanese varieties $\operatorname{Alb}_{\mathscr{F}}^{(i)}(X)(i=1,0)$ satisfy an obvious functoriality property (Proposition 2.22). 
Section 3 is the main part of this work, where we establish a higher-dimensional analogue to the generalized Jacobian with modulus of Rosenlicht and Serre. Let $X$ be a smooth proper variety over a perfect field $k$. We use the notion of modulus from [Kato and Russell 2010], which associates to a rational map $\varphi: X \rightarrow P$ an effective divisor $\bmod (\varphi)$ on $X$ (Definition 3.11). If $D$ is an effective divisor on $X$, we define a formal subgroup $\mathscr{F}_{X, D}=\left(\mathscr{F}_{X, D}\right)_{\text {ét }} \times_{k}\left(\mathscr{F}_{X, D}\right)_{\text {inf }}$ of $\underline{\operatorname{Div}}_{X}$ (Definition 3.14) by the conditions

$$
\left(\mathscr{F}_{X, D}\right)_{\text {ét }}=\left\{B \in \underline{\operatorname{Div}}_{X}(k) \mid \operatorname{Supp}(B) \subset \operatorname{Supp}(D)\right\},
$$

and for $\operatorname{char}(k)=0$,

$$
\left(\mathscr{F}_{X, D}\right)_{\text {inf }}=\exp \left(\widehat{\mathbb{G}}_{\mathrm{a}} \otimes_{k} \Gamma\left(X, \mathscr{O}_{X}\left(D-D_{\text {red }}\right) / \mathcal{O}_{X}\right)\right),
$$

and for $\operatorname{char}(k)=p>0$,

$$
\left(\mathscr{F}_{X, D}\right)_{\mathrm{inf}}=\operatorname{Exp}\left(\sum_{r>0}{ }_{r} \widehat{W} \otimes_{W(k)} \Gamma\left(X, \mathrm{fil}_{D-D_{\text {red }}}^{\mathrm{F}} W_{r}\left(\mathscr{K}_{X}\right) / W_{r}\left(\mathcal{O}_{X}\right)\right), 1\right),
$$

where $D_{\text {red }}$ is the underlying reduced divisor of $D$, Exp denotes the Artin-Hasse exponential, ${ }_{r} \widehat{W}$ is the kernel of the $r$-th power of the Frobenius on the completion $\widehat{W}$ of the Witt group $W$ at 0 and $\operatorname{fil}_{D}^{\mathrm{F}} W_{r}\left(\mathscr{K}_{X}\right)$ is a filtration of the Witt group (Definition 3.2). Let

$$
\mathscr{F}_{X, D}^{0, \text { red }}=\mathscr{F}_{X, D} \times \underline{\operatorname{Div}}_{X} \underline{\operatorname{Div}}_{X}^{0, \text { red }}
$$

be the intersection of $\mathscr{F}_{X, D}$ and $\underline{\operatorname{Div}}_{X}^{0, \text { red }}$. The formal groups $\mathscr{F}_{X, D}$ and $\mathscr{F}_{X, D}^{0, \text { red }}$ are dual-algebraic (Proposition 3.15).

Then $\bmod (\varphi) \leq D$ if and only if $\operatorname{im}\left(\tau_{\varphi}\right) \subset \mathscr{F}_{X, D}^{0, \text { red }}$ (Lemma 3.16). This yields (see Theorem 3.18 and Theorem 3.19):

Theorem 0.2. The category $\operatorname{Mr}(X, D)$ of those rational maps $\varphi: X \rightarrow P$ such that $\bmod (\varphi) \leq D$ admits a universal object $\operatorname{alb}_{X, D}^{(1)}: X \rightarrow \operatorname{Alb}^{(1)}(X, D)$, called the Albanese of $X$ of modulus $D$. The algebraic group $\operatorname{Alb}^{(0)}(X, D)$ acting on $\operatorname{Alb}^{(1)}(X, D)$ is dual to the 1 -motive $\left[\mathscr{F}_{X, D}^{0, \text { red }} \rightarrow \operatorname{Pic}_{X}^{0, \text { red }}\right]$.

The Albanese varieties with modulus $\operatorname{Alb}^{(i)}(X, D)$ for $i=1,0$ are functorial (Proposition 3.22). In the case that $X=C$ is a curve, our Albanese with modulus $\operatorname{Alb}^{(i)}(C, D)$ coincide with the generalized Jacobians with modulus $J^{(i)}(C, D)$ of Rosenlicht and Serre (Theorem 3.25 and Galois descent).

A relative Chow group $\mathrm{CH}_{0}(X, D)$ of modulus $D$ is introduced in Definition 3.27. We say a rational map $\varphi: X \rightarrow P$ to a torsor $P$ under a commutative algebraic group $G$ factors through $\mathrm{CH}_{0}(X, D)^{0}$ if the associated map $\mathrm{Z}_{0}(U)^{0} \rightarrow G(k)$, $\sum l_{i} p_{i} \mapsto \sum l_{i} \varphi\left(p_{i}\right)$ on 0 -cycles of degree 0 (where $U$ is the open set on which $\varphi$ is defined) factors through a homomorphism of abstract groups $\mathrm{CH}_{0}(X, D)^{0} \rightarrow G(k)$. We show (see Theorem 3.29): 
Theorem 0.3. Assume $k$ is algebraically closed. A rational map $\varphi: X \rightarrow P$ factors through $\mathrm{CH}_{0}(X, D)^{0}$ if and only if it factors through $\mathrm{Alb}^{(1)}(X, D)$. In other words, $\mathrm{Alb}^{(0)}(X, D)$ is a universal quotient of $\mathrm{CH}_{0}(X, D)^{0}$.

The theory of Albanese varieties with modulus has an application to the class field theory of function fields of varieties over finite fields. Let $X$ be a geometrically irreducible projective variety over a finite field $k=\mathbb{F}_{q}$. Let $\bar{k}$ be an algebraic closure of $k$. Let $\mathrm{K}_{X}$ denote the function field of $X$, and $\mathrm{K}_{X}^{\mathrm{ab}}$ be the maximal abelian extension of $\mathrm{K}_{X}$. From Lang's class field theory one obtains:

Theorem 0.4. The geometric Galois group $\mathrm{Gal}\left(\mathrm{K}_{X}^{\mathrm{ab}} / \mathrm{K}_{X} \bar{k}\right)$ is isomorphic to the projective limit of the $k$-rational points of the Albanese varieties of $X$ with modulus $D$

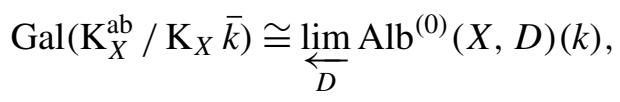

where $D$ ranges over all effective divisors on $X$ rational over $k$.

The proof of Theorem 0.4 is analogous to the proof of Lang's class field theory given in [Serre 1959, VI, §4, nos. 16-19], replacing maximal maps by the universal objects $\operatorname{alb}_{X, D}^{(1)}: X \rightarrow \operatorname{Alb}^{(1)}(X, D)$ for the category of rational maps to $k$-torsors of modulus $\leq D$ from Theorem 0.2.

\section{1-motives}

The aim of this section is to construct a category of generalized 1-motives that contains all smooth connected commutative algebraic groups over a perfect field and provides a notion of duality for them.

1.1. Algebraic groups and formal groups. I will use the language of group functors, algebraic groups and formal groups. References for algebraic groups are [Demazure and Gabriel 1970; Waterhouse 1979], and for formal groups and Cartier duality are [SGA3 1970, VII ${ }_{\mathrm{B}}$; Demazure 1972, II; Fontaine 1977, I].

By algebraic group and formal group I will always mean a commutative (algebraic and formal, respectively) group.

Let $k$ be a ring (that is, associative, commutative and with unit). Set denotes the category of sets, $\mathrm{Ab}$ the category of abelian groups. Alg/ $k$ denotes the category of $k$-algebras, and Art/ $k$ the category of finite $k$-algebras (that is, of finite length). A $k$-functor is by definition a covariant functor from Alg/ $k$ to Set. A formal $k$-functor is by definition a covariant functor from Art $/ k$ to Set. A (formal) $k$-functor with values in $\mathrm{Ab}$ is called a (formal) $k$-group functor.

A $k$-group (or $k$-group scheme) is by definition a $k$-group functor with values in $\mathrm{Ab}$ whose underlying set-valued $k$-functor is represented by a $k$-scheme. The category of $k$-groups is denoted by $\mathscr{G} / k$, and the category of affine $k$-groups by 
$\varphi_{a} / k$. An algebraic $k$-group (or just algebraic group) is a $k$-group whose underlying scheme is separated and of finite type over $k$. The category of algebraic $k$-groups is denoted by $a \varphi / k$, and the category of affine algebraic $k$-groups by $a \mathscr{G} a / k$.

Now let $k$ be a field. A formal $k$-scheme is by definition a formal $k$-functor with values in Set that is the limit of a directed inductive system of finite $k$-schemes. Let $\mathscr{A}$ be a profinite $k$-algebra. The formal spectrum of $\mathscr{A}$ is the formal $k$-functor that assigns to $R \in$ Art/ $k$ the set of continuous homomorphisms of $k$-algebras from the topological ring $\mathscr{A}$ to the discrete $\operatorname{ring} R: \operatorname{Spf} \mathscr{A}(R)=\operatorname{Hom}_{k \text {-alg }}^{\text {cont }}(\mathscr{A}, R)$.

A formal $k$-group (or just formal group) is a formal $k$-group functor with values in $\mathrm{Ab}$ whose underlying set-valued formal $k$-functor is represented by a formal $k$-scheme, or equivalently is isomorphic to $\operatorname{Spf} \mathscr{A}$ for some profinite $k$-algebra $\mathscr{A}$. The category of formal $k$-groups is denoted by $G_{f} / k$.

Remark 1.1. A formal $k$-group $\mathscr{F}:$ Art $/ k \rightarrow$ Ab extends in a natural way to a $k$-group functor $\widetilde{\mathscr{F}}: \mathrm{Alg} / k \rightarrow \mathrm{Ab}$, by defining $\widetilde{\mathscr{F}}(R)$ for $R \in \mathrm{Alg} / k$ as the inductive limit of the $\mathscr{F}(S)$, where $S$ ranges over the finite $k$-subalgebras of $R$. If $\mathscr{F}=\operatorname{Spf} \mathscr{A}$ for some profinite $k$-algebra $\mathscr{A}$, then $\widetilde{\mathscr{F}}(R)=\operatorname{Hom}_{k \text {-alg }}^{\text {cont }}(\mathscr{A}, R)$ for every $R \in \mathrm{Alg} / k$.

Theorem 1.2. A formal $k$-group $\mathscr{F}$ is canonically an extension of an étale formal $k$-group $\mathscr{F}_{\text {ét }}$ by an infinitesimal (= connected) formal $k$-group (that is, the formal spectrum of a local ring) $\mathscr{F}_{\text {inf. Here }}$

$$
\mathscr{F}_{\text {ét }}(R)=\mathscr{F}\left(R_{\text {red }}\right) \quad \text { and } \quad \mathscr{F}_{\text {inf }}(R)=\operatorname{ker}\left(\mathscr{F}(R) \rightarrow \mathscr{F}\left(R_{\text {red }}\right)\right)
$$

for $R \in \operatorname{Art} / k$, where $R_{\mathrm{red}}=R / \operatorname{Nil}(R)$. If the base field $k$ is perfect, there is a unique isomorphism $\mathscr{F}_{\mathscr{F}} \cong \mathscr{F}_{\text {inf }} \times_{k} \mathscr{F}_{\text {ét }}$.

Proof. See [Demazure 1972, I, No. 7, Proposition on p. 34] or [Fontaine 1977, I, 7.2, p. 46].

Let $R$ be a ring. An $R$-sheaf is a sheaf (of sets) on Alg/R for the topology fppf. An $R$-sheaf with values in Ab is called an $R$-group sheaf. The category of $R$-group sheaves is denoted by $A b / R$.

Let $k$ be a field. The category of $k$-groups $\varphi / k$ and the category of formal $k$-groups $\mathscr{G}_{f} / k$ are full subcategories of $\mathscr{A} b / k$. This can be seen as follows: A $k$-functor that is represented by a scheme is a sheaf; see [Demazure and Gabriel 1970, III, $\S 1,1.3]$. This gives the sheaf property for $\mathscr{G} / k$ by definition. For $\mathscr{G} f / k$ we can reduce to this case by Remark 1.1 and the fact that a formal $k$-group is the direct limit of finite $k$-schemes.

1.1.1. Linear group associated to a ring. Let $k$ be a field.

Definition 1.3. Let $R$ be a $k$-algebra. The linear group associated to $R$ is the Weil restriction $\mathbb{L}_{R}:=\Pi_{R / k} \mathbb{G}_{\mathrm{m}, R}:=\mathbb{G}_{\mathrm{m}}(\cdot \otimes R)$ of $\mathbb{G}_{\mathrm{m}, R}$ from $R$ to $k$. 
If $S$ is a finite $k$-algebra, then $\mathbb{Q}_{S}$ is an affine algebraic $k$-group, according to [Demazure and Gabriel 1970, I, §1, 6.6].

Lemma 1.4. Let $k$ be a perfect field. Every affine algebraic $k$-group $L$ is isomorphic to a closed subgroup of $\mathbb{\bigsqcup}_{S}$ for some $S \in A r t / k$.

Proof. By Galois descent we can reduce to the case that $k$ is algebraically closed. Every affine algebraic $k$-group $L$ is isomorphic to a closed subgroup of $\mathrm{GL}_{r}$ for some $r \in \mathbb{N}$; see [Waterhouse 1979, 3.4 Theorem, p. 25]. Let $\rho: L \rightarrow \mathrm{GL}_{r}$ be a faithful representation. Define $S$ to be the group algebra of $\rho(L)$, that is, the $k$-subalgebra of the algebra of $(r \times r)$-matrices $\operatorname{Mat}_{r \times r}(k)$ generated by $\rho(L)(k)$. In particular, $S$ is finite-dimensional. Here we may assume that $L$ is reduced, hence determined by its $k$-valued points; otherwise embed the multiplicative part into $\left(\mathbb{G}_{\mathrm{m}}\right)^{t}$ for some $t \in \mathbb{N}$ (see [Demazure and Gabriel 1970, IV, §1, 1.5]) and the unipotent part into $\left(W_{r}\right)^{n}$ for some $r, n \in \mathbb{N}$ (see [ibid., $\left.\mathrm{V}, \S 1,2.5\right]$ ), and replace $L$ by $\left(\mathbb{G}_{\mathrm{m}}\right)^{t} \times{ }_{k}\left(W_{r}\right)^{n}$. Then $\rho(L)(k)$ is contained in the unit group of $S$, and $\rho: L \rightarrow \mathbb{G}_{\mathrm{m}}(\cdot \otimes S)=\mathbb{L}_{S}$ is a monomorphism from $L$ to $\mathbb{L}_{S}$.

1.1.2. Cartier duality. Let $k$ be a field. We will use the functorial description of Cartier-duality as in [Demazure 1972, II, No. 4]. We may consider formal groups as objects of $A b / k$; see Remark 1.1. Let $G$ be a $k$-group sheaf. Let $\underline{\operatorname{Hom}}_{\nexists b / k}\left(G, \mathbb{G}_{\mathrm{m}}\right)$ be the $k$-group functor defined by $R \mapsto \operatorname{Hom}_{\Re b / R}\left(G_{R}, \mathbb{G}_{\mathrm{m}, R}\right)$, which assigns to a $k$-algebra $R$ the group of homomorphisms of $R$-group sheaves from $G_{R}$ to $\mathbb{G}_{\mathrm{m}, R}$.

Theorem 1.5. If $G$ is an affine group or formal group), the k-group functor $\underline{\operatorname{Hom}}_{\sharp b / k}\left(G, \mathbb{G}_{\mathrm{m}}\right)$ is represented by a formal group or affine group, respectively, $G^{\vee}$, which is called the Cartier dual of $G$.

Cartier duality is an antiequivalence between the category of affine groups $G_{a} / k$ and the category of formal groups $\mathscr{G}_{f} / k$. The functors $L \mapsto L^{\vee}$ and $\mathscr{F} \mapsto \mathscr{F}^{\vee}$ are quasiinverse to each other.

Proof. See [SGA3 1970, $\mathrm{VII}_{\mathrm{B}}, 2.2 .2$ ] or [Fontaine 1977, I, 5.4, p. 37] for a description of Cartier duality via bialgebras. See [Demazure 1972, II, No. 4, Theorem, p. 27] for one direction of the functorial description of Cartier duality (it is only one direction since formal groups and affine groups are not considered as objects of the same category there). According to Section 1.1 and the properties of the group functor $\underline{\operatorname{Hom}}_{\nexists b / k}\left(G, \mathbb{G}_{\mathrm{m}}\right)$ as described in [Demazure and Gabriel 1970, II, $\S 1,2.10]$, it is an easy exercise to invert the given direction $L \mapsto L^{\vee}$ of the functorial description (one has to replace affine groups by formal groups, $\otimes_{k}$ by $\widehat{\otimes}_{k}$, Hom $_{k \text {-alg }}^{\text {cont }}$ by Hom $_{k \text {-alg }}$ and Spf by Spec).

Lemma 1.6. Let $L$ be an affine group and $R$ a $k$-algebra. The $R$-valued points of the Cartier-dual of $L$ are given by $L^{\vee}(R)=\operatorname{Hom}_{\Im A b / k}\left(L, \mathbb{L}_{R}\right)$. 
Proof. The statement is due to the fact that Weil restriction is right-adjoint to base extension

$$
L^{\vee}(R)=\operatorname{Hom}_{\mathscr{A} b / R}\left(L_{R}, \mathbb{G}_{\mathrm{m}, R}\right)=\operatorname{Hom}_{\Re b / k}\left(L, \mathbb{G}_{\mathrm{m}, R}(\cdot \otimes R)\right) .
$$

Cartier dual of a multiplicative group.

Proposition 1.7 [Demazure 1972, II, No. 8]. Let L be an affine k-group. Then L is multiplicative if and only if the Cartier-dual $L^{\vee}$ is an étale formal k-group.

Example 1.8. In particular, the Cartier-dual of a split torus $T \cong\left(\mathbb{G}_{\mathrm{m}}\right)^{t}$ is a lattice of the same rank: $T^{\vee} \cong \mathbb{Z}^{t}$, that is, a torsion-free étale formal group.

Proposition 1.9 [Demazure and Gabriel 1970, IV, §1, 1.2]. Let L be a multiplicative $k$-group. Then $L$ is algebraic if and only if $L^{\vee}(\bar{k})$ is of finite type.

Cartier dual of a unipotent group.

Proposition 1.10 [Demazure 1972, II, No. 9]. Let $L$ be an affine $k$-group. Then $L$ is unipotent if and only if the Cartier-dual $L^{\vee}$ is an infinitesimal formal k-group.

Example 1.11 (Cartier duality of Witt vectors). Suppose $\operatorname{char}(k)=p>0$. Let $W$ denote the $k$-group of Witt-vectors, $W_{r}$ the $k$-group of Witt-vectors of finite length $r$. Let $\widehat{W}$ be the completion of $W$ at 0 , that is, $\widehat{W}$ is the subfunctor of $W$ that associates to $R \in \mathrm{Alg} / k$ the set of $\left(w_{0}, w_{1}, \ldots\right) \in W(R)$ such that $w_{\nu} \in \operatorname{Nil}(R)$ for all $v \in \mathbb{N}$ and $w_{v}=0$ for almost all $v \in \mathbb{N}$. Moreover let ${ }_{r} \widehat{W}=\operatorname{ker}\left(\mathrm{F}^{r}: \widehat{W} \rightarrow \widehat{W}^{\left(p^{r}\right)}\right)$ be the kernel of the $r$-th power of the Frobenius F. Let $\Lambda$ denote the affine $k$-group that associates with $R \in \mathrm{Alg} / k$ the multiplicative group $1+t R \llbracket t \rrbracket$ of formal power series in $R$. Let $\mathrm{E}$ be the series

$$
\mathrm{E}(t)=\exp \left(-\sum_{r \geq 0} \frac{t^{p^{r}}}{p^{r}}\right)=\prod_{\substack{r \geq 1 \\(r, p)=1}}\left(1-t^{r}\right)^{\mu(r) / r},
$$

where $\mu$ denotes the Möbius function. The Artin-Hasse exponential is the homomorphism of $k$-groups Exp : $W \rightarrow \Lambda$ defined by

$$
\operatorname{Exp}(w, t):=\operatorname{Exp}(w)(t):=\prod_{r \geq 0} \mathrm{E}\left(w_{r} t^{p^{r}}\right) .
$$

For details see for instance [Demazure 1972, III, Nos. 1 and 2].

Then $\widehat{W}$ and ${ }_{r} \widehat{W}$ are Cartier-dual to $W$ and $W_{r}$, respectively, and the pairings $\langle\cdot, \cdot\rangle: \widehat{W} \times W \rightarrow \mathbb{G}_{\mathrm{m}}$ and $\langle\cdot, \cdot\rangle:{ }_{r} \widehat{W} \times W_{r} \rightarrow \mathbb{G}_{\mathrm{m}}$ are given by

$$
\langle v, w\rangle=\operatorname{Exp}(v \cdot w, 1)=\prod_{\substack{r \geq 0 \\ s \geq 0}} \mathrm{E}\left(v_{r}^{p^{s}} w_{s}^{p^{r}}\right) .
$$

See [Demazure and Gabriel 1970, V, §4, Proposition 4.5 and Corollary 4.6]. 
Proposition 1.12. Suppose $\operatorname{char}(k)=p>0$. Let $L$ be an affine $k$-group. The following conditions are equivalent:

(i) L is unipotent algebraic.

(ii) There is a monomorphism $L \hookrightarrow\left(W_{r}\right)^{n}$ for some $r, n \in \mathbb{N}$.

(iii) There is an epimorphism $\left({ }_{r} \widehat{W}\right)^{n} \rightarrow L^{\vee}$ for some $r, n \in \mathbb{N}$.

(Here we use the notation from Example 1.11.)

Proof. (i) $\Longrightarrow$ (ii) [Demazure and Gabriel 1970, V, §1, 2.5].

(ii) $\Longrightarrow$ (i) The underlying $k$-scheme of $W_{r}$ is the affine space $\mathbb{A}^{r}$; thus $W_{r}$ is algebraic. $0=W_{0} \subset W_{1} \subset W_{2} \subset \cdots \subset W_{r}$ is a filtration of $W_{r}$ with quotients $W_{\nu} / W_{\nu-1} \cong W_{1}=\mathbb{G}_{\mathrm{a}}$; hence $W_{r}$ is unipotent, according to [ibid., IV, §2, 2.5]. Products of unipotent groups and closed subgroups of a unipotent group are unipotent by [ibid., IV, $\S 2,2.3]$. Since $L$ is isomorphic to a closed subgroup of $\left(W_{r}\right)^{n}$, it is unipotent and algebraic.

(ii) $\Longleftrightarrow$ (iii) This is due to Cartier duality of Witt vectors; see Example 1.11.

1.1.3. Dual abelian variety. Let $k$ be a field. Let $A$ be an abelian variety over $k$. The dual of $A$ is given by $A^{\vee}=\mathrm{Pic}^{0} A$. According to the generalized Barsotti-Weil formula (see [Oort 1966, III.18]), the dual abelian variety $A^{\vee}$ represents the $k$-group sheaf $\underline{\operatorname{Ext}}_{\mathscr{A} b / k}^{1}\left(A, \mathbb{G}_{\mathrm{m}}\right)$ associated to $R \mapsto \operatorname{Ext}_{\mathscr{A b} / R}^{1}\left(A_{R}, \mathbb{G}_{\mathrm{m}, R}\right)$.

Proposition 1.13. Let $A$ be an abelian $k$-variety and $S$ a finite $k$-algebra. There is a canonical isomorphism

$$
\operatorname{Ext}_{\mathscr{A} b / k}^{1}\left(A, \mathbb{L}_{S}\right) \stackrel{\sim}{\rightarrow} \operatorname{Ext}_{\mathscr{A b} / S}^{1}\left(A_{S}, \mathbb{G}_{\mathrm{m}, S}\right) .
$$

Thus the S-valued points of the dual abelian variety are given by

$$
A^{\vee}(S)=\operatorname{Ext}_{\Im a b / k}^{1}\left(A, \mathbb{L}_{S}\right) .
$$

Proof. Consider the following composition of functors on $\mathscr{A b} / S$ :

$$
\operatorname{Hom}_{\Re b / k}(A, \cdot) \circ \Pi_{S / k}: G \mapsto \operatorname{Hom}_{A b / k}(A, G(\cdot \otimes S))=\operatorname{Hom}_{\Re b / S}\left(A_{S}, G\right) .
$$

Since $\operatorname{Ext}_{\mathscr{A} b / k}^{1}\left(A, \mathbb{L}_{S}\right)$ and $\operatorname{Ext}_{\mathscr{A} b / S}^{1}\left(A_{S}, \mathbb{G}_{\mathrm{m}, S}\right)$ are identified with the sets of primitive elements in $\mathrm{H}^{1}\left(A, \mathbb{L}_{S}\left(\mathbb{O}_{A}\right)\right)$ and $\mathrm{H}^{1}\left(A_{S}, \mathbb{G}_{\mathrm{m}}\left(\mathbb{O}_{A_{S}}\right)\right.$ ), (see [Serre 1959, VII, no. 15, théorème 5] and [Oort 1966, III.17.6], respectively), we may compute these Extgroups using the étale site instead of the flat site, according to [Milne 1980, III, Theorem 3.9]. As $S$ is a finite $k$-algebra, the Weil restriction $\Pi_{S / k}: G \mapsto G(\cdot \otimes S)$ is exact for the étale topology (see [Milne 1980, II, Corollary 3.6]). Then the exact sequence of low degree terms of the Grothendieck spectral sequence yields a canonical isomorphism

$$
\left(\mathrm{R}^{1} \operatorname{Hom}_{\mathscr{A} b / k}(A, \cdot)\right)\left(\Pi_{S / k}\left(\mathbb{G}_{\mathrm{m}}\right)\right) \stackrel{\sim}{\longrightarrow} \mathrm{R}^{1}\left(\operatorname{Hom}_{\mathscr{A} b / k}(A, \cdot) \circ \Pi_{S / k}\right)\left(\mathbb{G}_{\mathrm{m}}\right)
$$


(see [Milne 1980, Appendix B, Corollary 2]), showing the statement.

1.1.4. Extensions of formal groups. Let $k$ be a field.

Lemma 1.14. $\underline{\operatorname{Ext}}_{\mathscr{A} b / k}^{1}\left(\mathscr{F}, \mathbb{G}_{\mathrm{m}}\right)=0$ for any dual-algebraic formal $k$-group $\mathscr{F}_{\text {. }}$

Proof. Let $L$ be the affine algebraic group dual to $\mathscr{F}$. Let $R$ be a $k$-algebra. We show that $\operatorname{Ext}_{\mathscr{A} b / R}^{1}\left(L_{R}^{\vee}, \mathbb{G}_{\mathrm{m}, R}\right)=0$ flat locally over $\operatorname{Spec} R$. As $L$ has a filtration $0=L_{0} \subset L_{1} \subset \cdots \subset L_{r}=L$ with quotients equal to $\mathbb{G}_{\mathrm{m}}, \mathbb{G}_{\mathrm{a}}$ or a finite $k$-group, it suffices to show the statement for $L=\mathbb{G}_{\mathrm{m}}, \mathbb{G}_{\mathrm{a}}$ or a finite group.

If $L$ is a finite $k$-group, the Cartier dual $L^{\vee}$ is again a finite $k$-group $F$, and $\operatorname{Ext}_{\mathscr{A b / R}}^{1}\left(F_{R}, \mathbb{G}_{\mathrm{m}, R}\right)=0$ flat locally according to [Milne 1980, III, §4, Lemma 4.17].

If $L=\mathbb{G}_{\mathrm{m}}$, then $L^{\vee}=\mathbb{Z}$, and $\operatorname{Ext}_{\mathscr{A} b / R}^{1}\left(\mathbb{Z}, \mathbb{G}_{\mathrm{m}}\right)=0$ is clear.

If $L=\mathbb{G}_{\mathrm{a}}$ and $\operatorname{char}(k)=0$, then $L^{\vee}=\widehat{\mathbb{G}}_{\mathrm{a}}$. We have

$$
\operatorname{Ext}_{\Re b / R}^{1}\left(\widehat{\mathbb{G}}_{\mathrm{a}}, \mathbb{G}_{\mathrm{m}}\right)=\operatorname{Ext}_{\Re \& / R}^{1}\left(\widehat{\mathbb{G}}_{\mathrm{a}}, \widehat{\mathbb{G}}_{\mathrm{a}}\right)=0 ;
$$

see [Barbieri-Viale and Bertapelle 2009, Lemma A.4.6].

If $L=\mathbb{G}_{\mathrm{a}}=W_{1}$ and $\operatorname{char}(k)=p>0$, then $L^{\vee}={ }_{1} \widehat{W}$ (with notation from Example 1.11). Since ${ }_{1} \widehat{W}=\operatorname{ker}(\mathrm{F}: \widehat{W} \rightarrow \widehat{W})$ is annihilated by the Frobenius $\mathrm{F}$ and since $\operatorname{ker}\left(\mathrm{F}: \mathbb{G}_{\mathrm{m}} \rightarrow \mathbb{G}_{\mathrm{m}}\right)=\mu_{p}$ is the group of $p$-th roots of unity over Spec $R$ (this group is finite, and hence both an algebraic group and a formal group), any extension $E \in \operatorname{Ext}_{\nexists b / R}^{1}\left({ }_{1} \widehat{W}, \mathbb{G}_{\mathrm{m}}\right)$ is the push-out of an extension $\mathscr{F}_{\mathcal{F}} \in \operatorname{Ext}_{\Re{ }^{\prime} / R}^{1}\left({ }_{1} \widehat{W}, \mu_{p}\right)$. As $\mu_{p}$ and ${ }_{1} \widehat{W}$ are base changes of formal $k$-groups, the affine algebra $\mathcal{O}\left(\mu_{p}\right)$ of $\mu_{p}$ is a free $R$-module of finite rank and the affine algebra $O\left({ }_{1} \widehat{W}\right)$ of ${ }_{1} \widehat{W}$ is the projective limit of free $R$-modules of finite rank; I will refer to those algebras as free profinite-rank $R$-algebras. The underlying $\mu_{p}$-bundle of $\mathscr{F}$ is flat locally trivial and hence flat locally the affine algebra of $\mathscr{F}$ is $\mathscr{O}(\mathscr{F})=\mathscr{O}\left(\mu_{p}\right) \otimes_{R} \mathcal{O}\left({ }_{1} \widehat{W}\right)$, and this is a free pro-finite-rank $R$-algebra as well. In this case Cartier duality works in the same way as for formal $k$-groups, ${ }^{1}$ so the exact sequence

$$
0 \rightarrow \mu_{p} \rightarrow \mathscr{F} \rightarrow{ }_{1} \widehat{W} \rightarrow 0
$$

is turned into the exact sequence

$$
0 \rightarrow \mathbb{G}_{\mathrm{a}} \rightarrow \mathscr{F}^{\vee} \rightarrow \mathbb{Z} / p \mathbb{Z} \rightarrow 0
$$

of $R$-groups, where $\mathscr{F}^{\vee}=\underline{\operatorname{Hom}}_{\mathscr{A b} / R}\left(\mathscr{F}, \mathbb{G}_{\mathrm{m}}\right)$. Applying $\underline{\operatorname{Hom}}_{\mathscr{A} / R}\left(\cdot, \mathbb{G}_{\mathrm{m}}\right)$ to the push-out diagram $\mathbb{G}_{\mathrm{m}} \leftarrow \mu_{p} \rightarrow \mathscr{F}_{\mathcal{F}}$ of $E$ shows that $E^{\vee}:=\underline{\operatorname{Hom}}_{A b / R}\left(E, \mathbb{G}_{\mathrm{m}}\right)$ is the pull-back of the diagram $\mathbb{Z} \rightarrow \mathbb{Z} / p \mathbb{Z} \leftarrow \mathscr{F}^{\vee}$. In particular, since $\mathscr{F}^{\vee} \rightarrow \mathbb{Z} / p \mathbb{Z}$ is surjective, $E^{\vee} \rightarrow \mathbb{Z}$ is surjective as well. Thus we obtain an exact sequence

$$
0 \rightarrow \mathbb{G}_{\mathrm{a}} \rightarrow E^{\vee} \rightarrow \mathbb{Z} \rightarrow 0,
$$

${ }^{1}$ The category of flat locally free pro-finite-rank $R$-algebras is not abelian. The references for Cartier duality listed in the proof of Theorem 1.5 make additional assumptions on the base ring $R$ in order to achieve that the category of $R$-formal groups is abelian. 
which is obviously split. Dualizing again gives the split exact sequence

$$
0 \rightarrow \mathbb{G}_{\mathrm{m}} \rightarrow E^{\vee \vee} \rightarrow{ }_{1} \widehat{W} \rightarrow 0
$$

where $E^{\vee \vee}=\underline{\operatorname{Hom}}_{A b / R}\left(E^{\vee}, \mathbb{G}_{\mathrm{m}}\right)$. The canonical map of any abelian sheaf $\mathscr{A}$ to its double dual $\mathscr{A}^{\vee \vee}$ yields the following commutative diagram with exact rows:

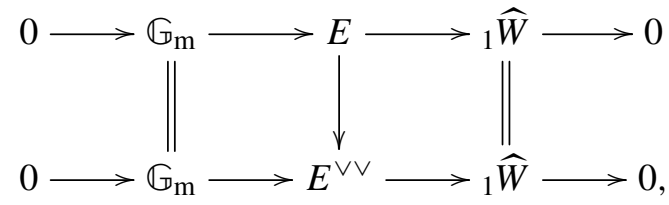

where the vertical arrow in the middle is an isomorphism by the Five Lemma. Thus $E \cong E^{\vee \vee}$ is split.

\subsection{1-motives with unipotent part. Let $k$ be a field.}

\subsubsection{Definition of a 1-motive with unipotent part.}

Definition 1.15. A formal $k$-group $\mathscr{F}$ is called dual-algebraic if its Cartier-dual $\mathscr{F}^{\vee}$ is algebraic. The category of dual-algebraic formal $k$-groups is denoted by $d \mathscr{G} f / k$.

Proposition 1.16. A formal $k$-group $\mathscr{F}$ is dual-algebraic if and only if the following conditions are satisfied:

(1) $\mathscr{F}(\bar{k})$ is of finite type,

(2) for $\operatorname{char}(k)=0$, Lie (F) is finite-dimensional, and for $\operatorname{char}(k)>0$, $\mathscr{F}_{\text {inf }}$ is a quotient of $\left({ }_{r} \widehat{W}\right)^{n}$ for some $r, n \in \mathbb{N}$ (see Example 1.11 for the definition of $r \widehat{W}$ ).

Proof. The decomposition of $\mathscr{F}$ into étale part and infinitesimal part gives the decomposition of the affine group $\mathscr{F}^{\vee}$ into multiplicative part and unipotent part, according to Propositions 1.7 and 1.10. Then that statement follows directly from Propositions 1.9 and 1.12 for $\operatorname{char}(k)>0$. For $\operatorname{char}(k)=0$, the claim in (2) follows since the Lie functor yields an equivalence between the category of commutative infinitesimal formal $k$-groups and the category of $k$-vector spaces; see [SGA3 1970, VII $\left._{\mathrm{B}}, 3.2 .2\right]$.

Lemma 1.17. Let F be a dual-algebraic formal group. Then any formal group $G$ that is a subgroup or a quotient of $\mathscr{F}$ is also dual-algebraic.

Proof. By Cartier-duality, this is equivalent to the dual statement about affine algebraic groups; see [Demazure 1972, II, No. 6, Corollary 4 of Theorem 2, p. 32].

Definition 1.18. A 1-motive with unipotent part is a tuple $M=(\mathscr{F}, L, A, G, \mu)$, where 
(a) $\mathscr{F}$ is a dual-algebraic formal group (Definition 1.15),

(b) $L$ is an affine algebraic group,

(c) $A$ is an abelian variety,

(d) $G$ is an extension of $A$ by $L$,

(e) $\mu: \mathscr{F} \rightarrow G$ is a homomorphism in $A b / k$.

A homomorphism between 1-motives with unipotent part $M=(\mathscr{F}, L, A, G, \mu)$ and $N=(\mathscr{E}, \Lambda, B, H, v)$ is a tuple $h=(\varphi, \lambda, \alpha, \gamma)$ of homomorphisms $\varphi: \mathscr{E} \rightarrow \mathscr{F}$, $\lambda: L \rightarrow \Lambda, \alpha: A \rightarrow B, \gamma: G \rightarrow H$, compatible with the structures of $M$ and $N$ as 1-motives with unipotent part, that is, giving an obvious commutative diagram.

For convenience, we will refer to a 1-motive with unipotent part only as a 1-motive.

If $G$ is a smooth connected algebraic group, it admits a canonical decomposition $0 \rightarrow L \rightarrow G \rightarrow A \rightarrow 0$ as an extension of an abelian variety $A$ by a connected affine algebraic group $L$, according to the theorem of Chevalley. Thus a homomorphism $\mu: \mathscr{F} \rightarrow G$ in $A b / k$ gives rise to a 1-motive $M=(\mathscr{F}, L, A, G, \mu)$ that we will denote just by $M=[\mathscr{F} \stackrel{\mu}{\rightarrow} G]$.

\subsubsection{Duality of 1-motives.}

Theorem 1.19. Let $L$ be an affine algebraic group and $A$ an abelian variety. There is a canonical isomorphism of abelian groups

$$
\Phi: \operatorname{Ext}_{\Im A b / k}^{1}(A, L) \stackrel{\sim}{\longrightarrow} \operatorname{Hom}_{\Re b / k}\left(L^{\vee}, A^{\vee}\right) .
$$

Proof. Consider the following left exact functor on $A b / k$ :

$$
\begin{aligned}
F: G \mapsto \operatorname{Bilin}_{\Re b l k}\left(A, L^{\vee} ; G\right) & =\operatorname{Hom}_{\Re b / k}\left(A, \underline{\operatorname{Hom}}_{\Re b / k}\left(L^{\vee}, G\right)\right) \\
& =\operatorname{Hom}_{\Re b l k}\left(L^{\vee}, \underline{\operatorname{Hom}}_{\Re b / k}(A, G)\right),
\end{aligned}
$$

where $\operatorname{Bilin}_{\mathscr{A} b / k}\left(A, L^{\vee} ; G\right)$ is the group of $\mathbb{Z}$-bilinear maps $A \times L^{\vee} \rightarrow G$ of sheaves of abelian groups. The two ways of writing $F$ as a composite yield the following two spectral sequences:

$$
\begin{aligned}
& \operatorname{Ext}_{\Re b / k}^{p}\left(A, \underline{\operatorname{Ext}}_{\& b / k}^{q}\left(L^{\vee}, G\right)\right) \Rightarrow \mathrm{R}^{p+q} F(G), \\
& \operatorname{Ext}_{\Re b / k}^{p}\left(L^{\vee}, \underline{\operatorname{Ext}}_{\Re b / k}^{q}(A, G)\right) \Rightarrow \mathrm{R}^{p+q} F(G) .
\end{aligned}
$$

For $G=\mathbb{G}_{\mathrm{m}}$, the associated exact sequences of low degree terms are

$$
0 \rightarrow \operatorname{Ext}^{1}\left(A, \underline{\operatorname{Hom}}\left(L^{\vee}, \mathbb{G}_{\mathrm{m}}\right)\right) \rightarrow \mathrm{R}^{1} F\left(\mathbb{G}_{\mathrm{m}}\right) \rightarrow \operatorname{Hom}\left(A, \underline{\operatorname{Ext}}^{1}\left(L^{\vee}, \mathbb{G}_{\mathrm{m}}\right)\right)=0,
$$


where the last term vanishes due to Lemma 1.14, and

$$
\begin{aligned}
0=\operatorname{Ext}^{1}\left(L^{\vee}, \underline{\operatorname{Hom}}\left(A, \mathbb{G}_{\mathrm{m}}\right)\right) \rightarrow \mathrm{R}^{1} F\left(\mathbb{G}_{\mathrm{m}}\right) \rightarrow & \operatorname{Hom}\left(L^{\vee}, A^{\vee}\right) \\
& \rightarrow \operatorname{Ext}^{2}\left(L^{\vee}, \underline{\operatorname{Hom}}\left(A, \mathbb{G}_{\mathrm{m}}\right)\right)=0 .
\end{aligned}
$$

Putting these together we obtain isomorphisms

$$
\operatorname{Ext}_{\mathscr{A} b / k}^{1}(A, L) \stackrel{\sim}{\longrightarrow} \mathrm{R}^{1} F\left(\mathbb{G}_{\mathrm{m}}\right) \stackrel{\sim}{\longrightarrow} \operatorname{Hom}_{\mathscr{A} b / k}\left(L^{\vee}, A^{\vee}\right)
$$

Remark 1.20 (explicit description of $\operatorname{Ext}^{1}(A, L) \stackrel{\sim}{\longrightarrow} \operatorname{Hom}\left(L^{\vee}, A^{\vee}\right)$ ). The isomorphism $\Phi$ in Theorem 1.19 sends $G \in \operatorname{Ext}^{1}(A, L)$ to the connecting homomorphism $\underline{\operatorname{Hom}}_{\Re b / k}\left(L, \mathbb{G}_{\mathrm{m}}\right) \rightarrow \underline{\operatorname{Ext}}_{\Re b / k}^{1}\left(A, \mathbb{G}_{\mathrm{m}}\right)$ in the long exact cohomology sequence obtained from applying $\underline{\operatorname{Hom}}_{A b / k}\left(\cdot, \mathbb{G}_{\mathrm{m}}\right)$ to the short exact sequence $0 \rightarrow L \rightarrow G \rightarrow A \rightarrow 0$. Explicitly, this is the map $\Phi(G): \lambda \mapsto \lambda_{*} G$, which sends $\lambda \in L^{\vee}(R)=\operatorname{Hom}_{A b / k}\left(L, \mathbb{L}_{R}\right)$ to the push-out $\lambda_{*} G \in \operatorname{Ext}_{\mathscr{A b l k}}^{1}\left(A, \mathbb{L}_{R}\right)=A^{\vee}(R)$ of the diagram $\mathbb{L}_{R} \stackrel{\lambda}{\leftarrow} L \rightarrow G$.

If $L=\mathbb{L}_{S}$ for some $S \in$ Art $/ k$, the inverse map of $\Phi$ is given by the map $\Phi^{-1}: \vartheta \mapsto \vartheta\left(\mathrm{id}_{L}\right)$, which sends a homomorphism $\vartheta: L^{\vee} \rightarrow A^{\vee}$ to the image $\vartheta\left(\operatorname{id}_{L}\right) \in \operatorname{Ext}_{\mathscr{A} l k}^{1}(A, L)=A^{\vee}(S)$ of the identity $\operatorname{id}_{L} \in \operatorname{Hom}_{\Re b / k}(L, L)=L^{\vee}(S)$. In general, a given homomorphism $\vartheta \in \operatorname{Hom}_{\mathscr{A} b / k}\left(L^{\vee}, A^{\vee}\right)$ can be written as an element $\left[L^{\vee} \rightarrow A^{\vee}\right] \in \mathscr{C}^{[-1,0]}(A b / k)$ of the category of two-term complexes in $A b / k$, with $L^{\vee}$ placed in degree -1 and $A^{\vee}$ in degree 0 . Then

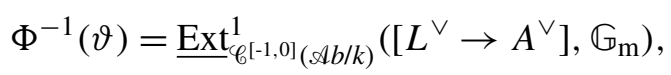

and this $k$-group sheaf is represented by an algebraic group: The short exact sequence $0 \rightarrow A^{\vee} \rightarrow\left[L^{\vee} \rightarrow A^{\vee}\right] \rightarrow L^{\vee}[1] \rightarrow 0$ gives rise to the exact sequence $0 \rightarrow \underline{\operatorname{Hom}}\left(L^{\vee}, \mathbb{G}_{\mathrm{m}}\right) \rightarrow \underline{\operatorname{Ext}^{1}}\left(\left[L^{\vee} \rightarrow A^{\vee}\right], \mathbb{G}_{\mathrm{m}}\right) \rightarrow \underline{\operatorname{Ext}^{1}}\left(A^{\vee}, \mathbb{G}_{\mathrm{m}}\right) \rightarrow 0$ since $\underline{\operatorname{Ext}}_{\mathscr{A b l k}}^{1}\left(L^{\vee}, \overline{\left.\mathbb{G}_{\mathrm{m}}\right)}=0\right.$ by Lemma 1.14. Thus $\underline{\operatorname{Ext}}_{\left.\mathscr{C}_{[}^{[-1,0]}(\mathscr{A}) / k\right)}^{1}\left(\left[L^{\vee} \rightarrow A^{\vee}\right], \mathbb{G}_{\mathrm{m}}\right)$ is an extension of $A$ by $L$.

Definition 1.21. The dual of a 1 -motive $M=(\mathscr{F}, L, A, G, \mu)$ is the 1 -motive $M^{\vee}=\left(L^{\vee}, \mathscr{F}^{\vee}, A^{\vee}, H, \eta\right)$, where

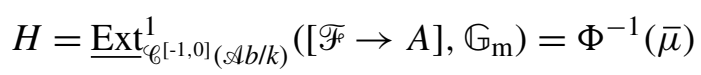

for $\bar{\mu}: \stackrel{\mu}{\mathscr{F}} \stackrel{\mu}{\rightarrow} G \rightarrow A$ the composite, and $\eta: L^{\vee} \rightarrow H$ the connecting homomorphism $\underline{\operatorname{Hom}}_{A b / k}\left(L, \mathbb{G}_{\mathrm{m}}\right) \rightarrow \underline{\operatorname{Ext}}_{\mathscr{C}^{[-1,0]}(\mathscr{A} / \mathrm{b} / k)}^{1}\left([\mathscr{F} \rightarrow A], \mathbb{G}_{\mathrm{m}}\right)$ in the long exact cohomology sequence associated with $0 \rightarrow[0 \rightarrow L] \rightarrow[\mathscr{F} \rightarrow G] \rightarrow[\mathscr{F} \rightarrow A] \rightarrow 0$.

Remark 1.22. The double dual $M^{\vee \vee}$ of a 1-motive $M$ is canonically isomorphic to $M$. If $M$ is of the form $[0 \rightarrow G]:=(0, L, A, G, 0)$, then the dual is

$$
\left[L^{\vee} \stackrel{\Phi(G)}{\longrightarrow} A^{\vee}\right]:=\left(L^{\vee}, 0, A^{\vee}, A^{\vee}, \Phi(G)\right) .
$$


If $M$ is of the form $[\mathscr{F} \stackrel{\mu}{\rightarrow} A]:=(\mathscr{F}, 0, A, A, \mu)$, the dual is

$$
\left[0 \rightarrow \Phi^{-1}(\mu)\right]:=\left(0, \mathscr{F}^{\vee}, A^{\vee}, \Phi^{-1}(\mu), 0\right) .
$$

This is clear by Theorem 1.19, and these "pure 1-motives" are the only ones that we are concerned with in this note. For the general case, the proof carries over literally from [Laumon 1996, (5.2.4)].

Proposition 1.23. Duality of 1-motives is functorial, that is, duality assigns to a homomorphism of 1-motives $h: M \rightarrow N$ a dual homomorphism $h^{\vee}: N^{\vee} \rightarrow M^{\vee}$.

Proof. Let $M=(\mathscr{F}, L, A, G, \mu)$ and $M^{\prime}=\left(\mathscr{F}^{\prime}, L^{\prime}, A^{\prime}, G^{\prime}, \mu^{\prime}\right)$ be 1-motives and $h: M \rightarrow M^{\prime}$ a homomorphism of 1-motives. Applying $\underline{\operatorname{Hom}}_{C_{[-1,0]}^{[}(A b / k)}\left(\cdot, \mathbb{G}_{\mathrm{m}}\right)$ to the commutative diagram with exact rows

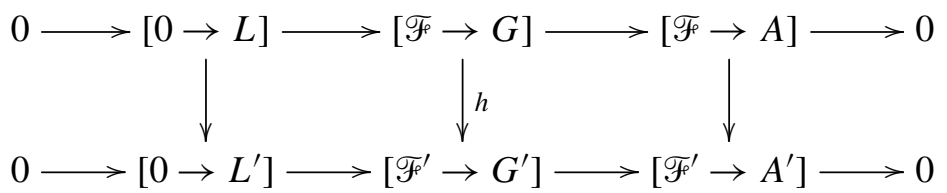

yields the homomorphism $h^{\vee}:\left[\left(L^{\prime}\right)^{\vee} \rightarrow H^{\prime}\right] \rightarrow\left[L^{\vee} \rightarrow H\right]$, where

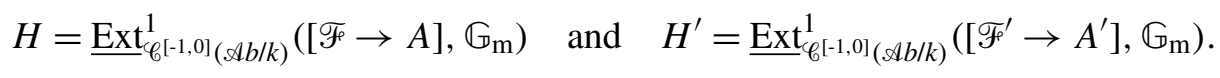

Applying $\underline{\operatorname{Hom}}_{\mathscr{C}^{[-1,0]}(\mathscr{A b} / k)}\left(\cdot, \mathbb{G}_{\mathrm{m}}\right)$ to the commutative diagram with exact rows

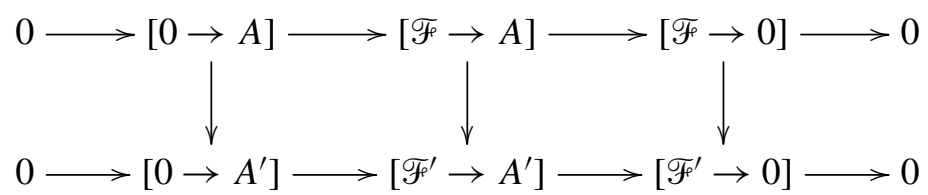

shows that the image of $\left(\mathscr{F}^{\prime}\right)^{\vee}$ under $h^{\vee}$ is contained in $\mathscr{F}^{\vee}$, which implies that $h^{\vee}:\left(M^{\prime}\right)^{\vee} \rightarrow M^{\vee}$ is a homomorphism of 1-motives.

\section{Universal rational maps}

The classical Albanese variety $\operatorname{Alb}(X)$ of a variety $X$ over a field $k$ (as in [Lang $1959, \mathrm{II}, \S 3])$ is an abelian variety, defined together with the Albanese map alb : $X \rightarrow \operatorname{Alb}(X)$ by the following universal mapping property: For every rational map $\varphi: X \rightarrow A$ to an abelian variety $A$ there is a unique homomorphism $h: \operatorname{Alb}(X) \rightarrow A$ such that $\varphi=h \circ$ alb up to translation by a constant $a \in A(k)$. Now we replace in this definition the category of abelian varieties by a subcategory $\mathrm{C}$ of the category of commutative algebraic groups. A result of Serre [1958-1959, No. 6, Théorème 8, p. 10-14] says that if the category $C$ contains the additive group $\mathbb{G}_{\mathrm{a}}$ and $X$ is a variety of dimension $>0$, there does not exist an Albanese variety in $C$ that is universal for all rational maps from $X$ to algebraic groups in C. One is therefore led to restrict 
the class of considered rational maps. This motivates the concept of categories of rational maps from $X$ to commutative algebraic groups, or more generally, categories of rational maps from $X$ to torsors under commutative algebraic groups (Definition 2.8), and to ask for the existence of universal objects for such categories.

For $k$ an algebraically closed field with $\operatorname{char}(k)=0$, in [Russell 2008, Section 2] I gave a criterion for which categories $\mathrm{Mr}$ of rational maps from a smooth proper variety $X$ over $k$ to algebraic groups there exists a universal object $\operatorname{Alb}_{\mathrm{Mr}}(X)$, as well as an explicit construction of these universal objects via duality of 1-motives. In this section we prove similar results for categories of rational maps, defined over a perfect field, from a smooth proper variety $X$ to torsors for commutative algebraic groups.

2.1. Relative Cartier divisors. The construction of such universal objects as above involves the functor $\underline{\operatorname{Div}}_{X}: \mathrm{Alg} / k \rightarrow \mathrm{Ab}$ of families of Cartier divisors, given by

$$
\underline{\operatorname{Div}}_{X}(R)=\left\{\begin{array}{l}
\text { Cartier divisors } \mathscr{D} \text { on } X \times_{k} \text { Spec } R \\
\text { whose fibers } \mathscr{D}_{p} \text { define Cartier divisors on } X \times_{k}\{p\} \\
\text { for all } p \in \operatorname{Spec} R
\end{array}\right\}
$$

for each $k$-algebra $R$, and for a homomorphism $h: R \rightarrow S$ in Alg/ $k$ the induced homomorphism $\underline{\operatorname{Div}}_{X}(h): \underline{\operatorname{Div}}_{X}(R) \rightarrow \underline{\operatorname{Div}}_{X}(S)$ in Ab is the pull-back of Cartier divisors on $X \times_{k} \operatorname{Spec} R$ to those on $X \times_{k} \operatorname{Spec} S$. The elements of $\underline{\operatorname{Div}}_{X}(R)$ are called relative Cartier divisors. See [Russell 2008, No. 2.1] for more details on $\underline{\operatorname{Div}}_{X}$.

We will be mainly concerned with the completion $\widehat{\operatorname{Div}}_{X}:$ Art $/ k \rightarrow A b$ of $\underline{\operatorname{Div}}_{X}$, which is given for every finite $k$-algebra $R$ by

$$
\widehat{\operatorname{Div}}_{X}(R)=\Gamma\left(X \otimes R,\left(\mathscr{K}_{X} \otimes_{k} R\right)^{*} /\left(\mathcal{O}_{X} \otimes_{k} R\right)^{*}\right) .
$$

We will regard $\widehat{\operatorname{Div}}_{X}$ as a subsheaf of $\underline{\operatorname{Div}}_{X}$; see Remark 1.1.

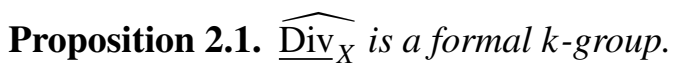

Proof. According to [Demazure 1972, I, No. 6; Fontaine 1977, I, §4] it suffices to show that $\widehat{\mathrm{Div}_{X}}$ is left-exact (that is, commutes with finite projective limits). We are going to show that $\widehat{\operatorname{Div}_{X}}$ is the composition of left-exact functors.

Let $R$ be a finite $k$-algebra. $\widehat{\operatorname{Div}_{X}}(R)=\Gamma(X, \mathscr{2}(R))$ is the abelian group of global sections of the sheaf $2(R):=\left(\operatorname{pr}_{X}\right)_{*}\left(\left(\mathscr{K}_{X} \otimes R\right)^{*} /\left(\mathcal{O}_{X} \otimes R\right)^{*}\right)$, where $\operatorname{pr}_{X}: X \otimes R \rightarrow X$ is the projection. The global section functor $\Gamma(X, \cdot)$ is known to be left-exact. We show that the formal $k$-group functor $2:$ Art $/ k \rightarrow A b / X$ (with values in the category of sheaves of abelian groups over $X$ ) commutes with finite projective limits (hence is left-exact): 
Let $\left(R_{i}\right)$ be a projective system of local finite $k$-algebras, with homomorphisms $h_{i j}: R_{j} \rightarrow R_{i}$ for $i<j$. We have projections $\operatorname{pr}_{j}: \lim _{\leftarrow} R_{i} \rightarrow R_{j}$ for each $j$, which commute with the $h_{i j}$. Functoriality of 2 in $R \in \overleftarrow{\text { Art }} / k$ induces homomorphisms $2\left(h_{i j}\right): 2\left(R_{j}\right) \rightarrow 2\left(R_{i}\right)$ and $2\left(\mathrm{pr}_{j}\right): 2\left(\lim _{\leftarrow} R_{i}\right) \rightarrow 2\left(R_{j}\right)$, which commute. The universal property of $\lim 2\left(R_{i}\right)$ yields a unique homomorphism of sheaves $2\left(\lim R_{i}\right) \rightarrow \lim 2\left(R_{i}\right)$. A homomorphism of sheaves is an isomorphism if and only if it is an isomorphism on stalks. Therefore it remains to show that the stalks $\mathscr{2}_{q}:$ Art $/ k \rightarrow$ Ab for $q \in X$ are left-exact in $R \in$ Art $/ k$. We have

$$
\mathscr{2}_{q}=\mathbb{G}_{\mathrm{m}}\left(\mathscr{K}_{X, q} \otimes_{k} \cdot\right) / \mathbb{G}_{\mathrm{m}}\left(\mathbb{O}_{X, q} \otimes_{k} \cdot\right) .
$$

The tensor product over a field $\mathscr{A} \otimes_{k} \cdot:$ Art $/ k \rightarrow A l g / k$ is exact for any $k$-algebra $\mathscr{A}$. Also the sheaf $\mathbb{G}_{\mathrm{m}}: \mathrm{Alg} / k \rightarrow \mathrm{Ab}$ is left-exact. Therefore the formal $k$-group functors $\mathbb{G}_{\mathrm{m}}\left(\mathscr{K}_{X, q} \otimes_{k} \cdot\right)$ and $\mathbb{G}_{\mathrm{m}}\left(\mathbb{O}_{X, q} \otimes_{k} \cdot\right)$ are formal $k$-groups. Since the category $\mathscr{G}_{f} / k$ of formal $k$-groups is abelian (see [SGA3 1970, $\left.\mathrm{VII}_{\mathrm{B}}, 2.4 .2\right]$ ), the quotient $2_{q}$ of these two formal $k$-groups is again a formal $k$-group.

Definition 2.2. Let $R$ be a finite $k$-algebra. If $D \in\left(\widehat{\widehat{\operatorname{Div}}_{X}}\right)$ ét $(R)$, then $\operatorname{Supp}(D)$ denotes the locus of zeroes and poles of local sections $\left(f_{\alpha}\right)_{\alpha}$ of $\mathbb{G}_{\mathrm{m}}\left(\mathscr{K}_{X} \otimes R_{\text {red }}\right)$ representing

$$
D \in \Gamma\left(\mathbb{G}_{\mathrm{m}}\left(\mathscr{K}_{X} \otimes R_{\mathrm{red}}\right) / \mathbb{G}_{\mathrm{m}}\left(\mathcal{O}_{X} \otimes R_{\mathrm{red}}\right)\right) .
$$

If $\delta \in\left(\widehat{\widehat{\operatorname{Div}}_{X}}\right)_{\text {inf }}(R)$, then $\operatorname{Supp}(\delta)$ denotes the locus of poles of local sections $\left(g_{\alpha}\right)_{\alpha}$ of $\mathbb{U}_{R}\left(\mathscr{K}_{X}\right)$ representing $\delta \in \Gamma\left(\mathbb{U}_{R}\left(\mathscr{K}_{X}\right) / \mathbb{U}_{R}\left(\mathbb{O}_{X}\right)\right)=\left(\widehat{\operatorname{Div}_{X}}\right)$ inf $(R)$, where $\mathbb{U}_{R}=$ $\operatorname{ker}\left(\mathbb{G}_{\mathrm{m}}(\cdot \otimes R) \rightarrow \mathbb{G}_{\mathrm{m}}\left(\cdot \otimes R_{\text {red }}\right)\right)$ is the unipotent part of $\mathbb{L}_{R}$ from Section 1.1.1

Definition 2.3. Let $\mathscr{F}_{F}$ be a formal subgroup of $\underline{\operatorname{Div}}_{X}$. The support of $\mathscr{F}$ is defined to be

$$
\operatorname{Supp}(\mathscr{F})=\bigcup_{\substack{R \in \operatorname{Art} / k \\ \mathscr{D} \in \mathscr{F}(R)}} \operatorname{Supp}(\mathscr{D})
$$

where we use the decomposition $\mathscr{F}_{F}=\mathscr{F}_{\text {ét }} \times \mathscr{F}_{\text {inf }}$ and Definition 2.2.

Suppose now that $X$ is a geometrically irreducible smooth proper variety over a perfect field $k$. Then the Picard functor $\underline{\mathrm{Pic}}_{X}$ is represented by a separated algebraic space $\mathrm{Pic}_{X}$, whose identity component $\overline{\mathrm{Pic}}_{X}^{0}$ is a proper scheme over $k$ (see [Bosch et al. 1990, No. 8.4, Theorem 3]). The underlying reduced scheme $\mathrm{Pic}_{X}^{0, \text { red }}$ of $\mathrm{Pic}_{X}^{0}$ is an abelian variety, called the Picard variety of $X$. The subfunctor of $\underline{P i c}_{X}$ that is represented by $\mathrm{Pic}_{X}^{0, \text { red }}$ will be denoted by $\underline{\mathrm{Pic}}_{X}^{0, \text { red }}$.

There is a natural transformation

$$
\mathrm{cl}: \underline{\operatorname{Div}}_{X} \rightarrow \underline{\operatorname{Pic}}_{X}
$$


We define $\underline{\operatorname{Div}}_{X}^{0, \text { red }}$ to be the subfunctor of $\underline{\operatorname{Div}}_{X}$ given by

$$
\underline{\operatorname{Div}}_{X}^{0, \text { red }}=\underline{\operatorname{Div}}_{X} \times_{\underline{\operatorname{Pic}}_{X}} \underline{\operatorname{Pic}}_{X}^{0, \text { red }} .
$$

2.2. Categories of rational maps to torsors. Let $X$ be a smooth proper variety over a perfect field $k$. Let $\bar{k}$ be an algebraic closure of $k$. In this note, algebraic groups and formal groups are commutative by definition (Section 1.1), and torsors are always torsors for commutative algebraic groups.

2.2.1. Induced transformation. Let $G$ be a smooth connected algebraic group, and let $0 \rightarrow L \rightarrow G \rightarrow A \rightarrow 0$ be the canonical decomposition of $G$, where $A$ is an abelian variety and $L$ an affine smooth connected algebraic group (theorem of Chevalley). Write $L=T \times_{k} U$ where $T$ is a torus and $U$ is unipotent; see [SGA3 1970, XVII, 7.2.1]. If $k$ is algebraically closed, $T \cong\left(\mathbb{G}_{\mathrm{m}}\right)^{t}$ for some $t \in \mathbb{N}$. If $k$ is of characteristic 0 , one has $U \cong\left(\mathbb{G}_{\mathrm{a}}\right)^{s}$ for some $s \in \mathbb{N}$ [Demazure and Gabriel 1970, $\mathrm{IV}, \S 2,4.2]$. If $k$ is of characteristic $p>0$, the unipotent group $U$ is embedded into a finite direct sum $\left(W_{r}\right)^{s}$ of Witt vector groups for some $r, s \in \mathbb{N}$ [ibid., $\left.\mathrm{V}, \S 1,2.5\right]$.

Since $\mathrm{H}_{\mathrm{fppf}}^{1}\left(\operatorname{Spec}\left(\mathbb{O}_{X, q}\right), \mathbb{G}_{\mathrm{m}}\right)=0$ and $\mathrm{H}_{\mathrm{fppf}}^{1}\left(\operatorname{Spec}\left(\mathbb{O}_{X, q}\right), U\right)=0$ for any point $q$ of $X$, we have exact sequences

$$
\begin{gathered}
0 \rightarrow L\left(\mathscr{K}_{X, q}\right) \rightarrow G\left(\mathscr{K}_{X, q}\right) \rightarrow A\left(\mathscr{K}_{X, q}\right) \rightarrow 0, \\
0 \rightarrow L\left(\mathscr{O}_{X, q}\right) \rightarrow G\left(\mathscr{O}_{X, q}\right) \rightarrow A\left(\mathscr{O}_{X, q}\right) \rightarrow 0 .
\end{gathered}
$$

Since a rational map to an abelian variety is defined at every smooth point (see [Lang 1959, II, §1, Theorem 2]), we have $A\left(\mathscr{K}_{X, q}\right)=A\left(\mathcal{O}_{X, q}\right)$ for every point $q$ of $X$. Hence the canonical map

$$
L\left(\mathcal{K}_{X, q}\right) / L\left(\mathcal{O}_{X, q}\right) \rightarrow G\left(\mathcal{K}_{X, q}\right) / G\left(\mathcal{O}_{X, q}\right)
$$

is bijective. By Cartier-duality, we have a pairing

$$
\langle\cdot, \cdot\rangle: L^{\vee} \times \Gamma\left(L\left(\mathscr{K}_{X}\right) / L\left(\mathbb{O}_{X}\right)\right) \rightarrow \Gamma\left(\mathbb{G}_{\mathrm{m}}\left(\underline{\mathscr{K}_{X}}\right) / \mathbb{G}_{\mathrm{m}}\left(\underline{\mathbb{O}_{X}}\right)\right),
$$

where $\underline{\mathscr{K}_{X}}:=\mathscr{K}_{X} \otimes \cdot$ and $\underline{\mathrm{O}_{X}}:=\mathrm{O}_{X} \otimes \cdot$.

Definition 2.4. Let $\varphi: X \rightarrow G$ be a rational map to a smooth connected algebraic group $G$, let $L$ be the affine part of $G$. Then $\tau_{\varphi}: L^{\vee} \rightarrow \widehat{\underline{\operatorname{Div}}_{X}}$ denotes the induced transformation given by $\left\langle\cdot, \ell_{\varphi}\right\rangle$, where $\ell_{\varphi}$ is the image of $\varphi \in G\left(\mathscr{K}_{X}\right)$ in $\Gamma\left(G\left(\mathscr{K}_{X}\right) / G\left(\mathcal{O}_{X}\right)\right) \stackrel{\sim}{\longrightarrow} \Gamma\left(L\left(\mathscr{K}_{X}\right) / L\left(\mathcal{O}_{X}\right)\right)$. By construction, $\tau_{\varphi}$ is a homomorphism of formal $k$-group functors.

Lemma 2.5. Let $G$ be a smooth connected algebraic group, let $L$ be the affine part of $G$. Let $\varphi: X \rightarrow G$ be a rational map. Let $\tau_{\varphi}: L^{\vee} \rightarrow \widehat{\text { Div }_{X}}$ be the induced transformation. Then $\operatorname{im}\left(\tau_{\varphi}\right)$ is a dual-algebraic formal group. 
Proof. $\widehat{\mathrm{Div}_{X}}$ is a formal group by Proposition 2.1, and $\varphi_{f} / k$ is a full subcategory of $\operatorname{Fctr}(\operatorname{Art} / k, \mathrm{Ab})$. Therefore $\tau_{\varphi}: L^{\vee} \rightarrow \widehat{\mathrm{Div}_{X}}$ is a homomorphism of formal groups. Since $\varphi_{f} / k$ is an abelian category, kernel and image of the homomorphism $\tau_{\varphi}$ are formal groups. Since $L$ is algebraic, $L^{\vee}$ is dual-algebraic and hence $\operatorname{im}\left(\tau_{\varphi}\right)$, as a quotient of $L^{\vee}$, is dual-algebraic (Lemma 1.17).

Lemma 2.6. Let $G \in \operatorname{Ext}_{\mathscr{A} b / k}^{1}(A, L)$ be a smooth connected algebraic group. Let $\varphi: X \rightarrow G$ be a rational map. Let $\tau_{\varphi}: L^{\vee} \rightarrow \widehat{\underline{\operatorname{Div}}_{X}}$ be the induced transformation. Then $\operatorname{im}\left(\tau_{\varphi}\right)$ is contained in the completion of $\underline{\operatorname{Div}}_{X}^{0, r e d}$.

Proof. As $A$ is an abelian variety, the composition $X \stackrel{\varphi}{\rightarrow} G \stackrel{\rho}{\rightarrow} A$ extends to a morphism $\bar{\varphi}: X \rightarrow A$. The description of the induced transformation $\tau_{\varphi}$ in terms of local sections into principal fiber bundles as given in [Russell 2008, No. 2.2] shows that the composition

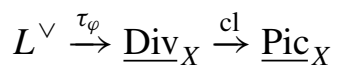

is given by $\lambda \mapsto \lambda_{*} G_{X}$, where $\lambda_{*} G$ is the push-out of $G \in \operatorname{Ext}_{\mathscr{A} b / k}^{1}(A, L)$ via $\lambda \in L^{\vee}(R)=\operatorname{Hom}_{\mathscr{A} b / k}\left(L, \mathbb{L}_{R}\right)$, and $G_{X}=G \times_{A} X$ is the fiber-product of $G$ and $X$ over $A$. Hence it comes down to showing that for each $R \in \operatorname{Art} / k$, each $\lambda \in L^{\vee}(R)$ the $\mathbb{L}_{R}$-bundle $\lambda_{*} G_{X}$ yields an element of $\operatorname{Pic}_{X}^{0, \text { red }}(R)$.

The universal mapping property of the classical Albanese $\operatorname{Alb}(X)$ yields that $\bar{\varphi}$ factors through $\operatorname{Alb}(X)$. Hence the pull-back $G_{X}=G \times_{A} X$ over $X$ is a pull-back of $G_{\mathrm{Alb}}=G \times{ }_{A} \operatorname{Alb}(X)$ over $\operatorname{Alb}(X)$. Then for each $\lambda \in L^{\vee}(R)$ the $\mathbb{L}_{R}$-bundle $\lambda_{*} G_{\mathrm{Alb}}$ over $\operatorname{Alb}(X)$ is an element of $\operatorname{Ext}_{\mathscr{A b l k}}^{1}\left(\operatorname{Alb}(X), \mathbb{L}_{R}\right)$, and hence gives an element of $\operatorname{Pic}_{\operatorname{Alb}(X)}^{0}(R)$. Since $\operatorname{Alb}(X)=\left(\operatorname{Pic}_{X}^{0, \text { red }}\right)^{\vee}$ is the dual abelian variety of $\mathrm{Pic}_{X}^{0, \text { red }}$, we have an isomorphism

$$
\operatorname{Pic}_{\operatorname{Alb}(X)}^{0} \stackrel{\sim}{\rightarrow} \operatorname{Pic}_{X}^{0, \text { red }}, \quad P \mapsto P_{X}=P \times_{\operatorname{Alb}(X)} X .
$$

As $\lambda_{*} G_{X}=\lambda_{*} G_{\mathrm{Alb}} \times \operatorname{Alb}(X) X$, we have $\lambda_{*} G_{X} \in \operatorname{Pic}_{X}^{0, \text { red }}(R)$.

Lemma 2.7. Let $L$ be an affine algebraic group and $\tau: L^{\vee} \rightarrow \widehat{\text { Div }_{X}^{0, r e d}}$ a homomorphism of formal groups. Let $G \in \operatorname{Ext}_{\$ b l k}^{1}(\operatorname{Alb}(X), L)$ be the extension corresponding to

$$
\mathrm{cl} \circ \tau: L^{\vee} \rightarrow \underline{\operatorname{Div}}_{X}^{0, \text { red }} \rightarrow \underline{\operatorname{Pic}}_{X}^{0, \text { red }}
$$

under the bijection $\Phi$ from Theorem 1.19. There is a rational map $\varphi: X \rightarrow G$ whose induced transformation is $\tau$, and $\varphi$ is determined uniquely up to translation by a constant $g \in G(k)$.

Proof. By Lemma 1.4 we may choose an embedding $\lambda: L \hookrightarrow \mathbb{L}_{S}$ for some finite ring $S \in$ Art $/ k$. Let $\mathscr{D} \in \underline{\operatorname{Div}}_{X}^{0, \text { red }}(S)$ be the image of $\operatorname{id}_{\mathbb{L}_{S}} \in \operatorname{Hom}_{A b / k}\left(\mathbb{L}_{S}, \mathbb{L}_{S}\right)=\mathbb{L}_{S}^{\vee}(S)$ under the composition $\tau \circ \lambda^{\vee}: \mathbb{L}_{S}^{\vee} \rightarrow L^{\vee} \rightarrow \underline{\text { Div }}_{X}$. Remark 1.20 shows that 
$\mathcal{O}_{X \otimes S}(\mathscr{D}) \in \operatorname{Pic}_{X}^{0, \text { red }}(S)$ is the line bundle corresponding to $G \in \operatorname{Ext}_{\mathscr{A} b / k}^{1}(\operatorname{Alb}(X), L)$ under the map

$$
\operatorname{Ext}_{\Im b / k}^{1}(\operatorname{Alb}(X), L) \rightarrow \operatorname{Ext}_{\Im b l k}^{1}\left(\operatorname{Alb}(X), \mathbb{L}_{S}\right)=\operatorname{Pic}_{\operatorname{Alb}(X)}^{0}(S) \stackrel{\sim}{\rightarrow} \operatorname{Pic}_{X}^{0, \text { red }}(S) .
$$

Let $\mathrm{G}_{\mathbb{L}_{S}}(\mathscr{D})$ be the image of $G$ in $\operatorname{Ext}_{\Re b / k}^{1}\left(\operatorname{Alb}(X), \mathbb{L}_{S}\right)$. Then the fiber product $\mathrm{P}_{\mathbb{L}_{S}}(\mathscr{D}):=\mathrm{G}_{\mathbb{L}_{S}}(\mathscr{D}) \times_{\operatorname{Alb}(X)} X$ is the $\mathbb{L}_{S}$-bundle on $X$ associated to $\mathscr{O}_{X \otimes S}(\mathscr{D})$, and $G$ and $P:=G \times \operatorname{Alb(X)} X$ are reductions of the $\mathbb{L}_{S}$-bundles $\mathrm{G}_{\mathbb{L}_{S}}(\mathscr{D})$ and $\mathrm{P}_{\mathbb{L}_{S}}(\mathscr{D})$ to the fiber $L$. The canonical 1-section of $0_{X \otimes S}(\mathscr{D})$ yields a section $X \rightarrow P$, and composition with $P \rightarrow G$ yields the desired rational map $\varphi: X \rightarrow G$, which by construction satisfies $\tau_{\varphi}=\tau$. Then

$$
\begin{aligned}
\ell_{\varphi} \in \Gamma\left(G\left(\mathscr{K}_{X}\right) / G\left(\mathscr{O}_{X}\right)\right) & \cong \Gamma\left(L\left(\mathscr{K}_{X}\right) / L\left(\mathscr{O}_{X}\right)\right) \\
& \subset \Gamma\left(\mathbb{L}_{S}\left(\mathscr{K}_{X}\right) / \mathbb{L}_{S}\left(\mathscr{O}_{X}\right)\right)=\Gamma\left(\mathbb{G}_{\mathrm{m}}\left(\mathscr{K}_{X} \otimes S\right) / \mathbb{G}_{\mathrm{m}}\left(\mathbb{O}_{X} \otimes S\right)\right)
\end{aligned}
$$

corresponding to $\mathscr{D}$ is uniquely determined by $\tau$. The rational map $\varphi \in G\left(\mathscr{K}_{X}\right)$, as a lift of $\ell_{\varphi}$, is determined up to a constant $g \in G(k)=\Gamma\left(G\left(0_{X}\right)\right)$, according to the exact sequence $0 \rightarrow \Gamma\left(G\left(\mathscr{O}_{X}\right)\right) \rightarrow \Gamma\left(G\left(\mathscr{K}_{X}\right)\right) \rightarrow \Gamma\left(G\left(\mathscr{K}_{X}\right) / G\left(\mathscr{O}_{X}\right)\right)$.

\subsubsection{Definition of a category of rational maps.}

Definition 2.8. A category Mr of rational maps from $X$ to torsors is a category satisfying the following conditions: The objects of $\mathrm{Mr}$ are rational maps $\varphi: X \rightarrow P$, where $P$ is a torsor for a smooth connected algebraic group. The morphisms of $\mathrm{Mr}$ between two objects $\varphi: X \rightarrow P$ and $\psi: X \rightarrow Q$ are given by the set of those homomorphisms of torsors $h: P \rightarrow Q$ such that $h \circ \varphi=\psi$.

Remark 2.9. Let $\varphi: X \rightarrow P$ and $\psi: X \rightarrow Q$ be two rational maps from $X$ to torsors. Then Definition 2.8 implies that for any category Mr of rational maps from $X$ to torsors containing $\varphi$ and $\psi$ as objects the set of morphisms $\operatorname{Hom}_{\mathrm{Mr}}(\varphi, \psi)$ is the same. Therefore two categories $\mathrm{Mr}$ and $\mathrm{Mr}^{\prime}$ of rational maps from $X$ to torsors are equivalent if every object of $\mathrm{Mr}$ is isomorphic to an object of $\mathrm{Mr}^{\prime}$.

Remark 2.10. If a $k$-torsor $P$ for an algebraic $k$-group $G$ admits a $k$-rational point, then $P$ may be identified with $G$. Thus for a rational map $\varphi: X \rightarrow P$ it makes sense to consider the base changed map $\varphi \otimes_{k} \bar{k}: X \otimes_{k} \bar{k} \rightarrow P \otimes_{k} \bar{k}$ as a rational map from $X \otimes_{k} \bar{k}=\bar{X}$ to an algebraic $\bar{k}$-group $P \otimes_{k} \bar{k} \cong G \otimes_{k} \bar{k}$.

Definition 2.11. The category of rational maps from $X$ to abelian varieties is denoted by Mav.

Remark 2.12. The objects of Mav are in fact morphisms from $X$ to abelian varieties, since a rational map from a smooth variety $X$ to an abelian variety $A$ extends to a morphism from $X$ to $A$; see [Lang 1959, II, §1, Theorem 2]. 
Definition 2.13. Let $\mathscr{F}$ be a dual-algebraic formal $k$-subgroup of $\underline{\operatorname{Div}}_{X}$. If $k$ is algebraically closed, then $\operatorname{Mr}_{\mathscr{F}}(X)$ denotes the category of all those rational maps $\varphi: X \rightarrow G$ from $X$ to algebraic $k$-groups for which the image of the induced transformation $\tau_{\varphi}: L^{\vee} \rightarrow \underline{\operatorname{Div}}_{X}$ (Definition 2.4) lies in $\mathscr{F}$, that is, which induce a homomorphism of formal groups $L^{\vee} \rightarrow \mathscr{F}$, where $L$ is the affine part of $G$. For general $k, \operatorname{MrF}_{\mathscr{F}}(X)$ denotes the category of all those rational maps $\varphi: X \rightarrow P$ from $X$ to $k$-torsors for which the base changed map $\varphi \otimes \bar{k}$ is an object of $\operatorname{Mr}_{\mathscr{F} \otimes \bar{k}}$. (Here we use Remark 2.10.)

2.3. Universal objects. Let $X$ be a smooth proper variety over a perfect field $k$. Algebraic groups are always assumed to be smooth and connected, and torsors are those for smooth connected algebraic groups, unless stated otherwise.

\subsubsection{Existence and construction.}

Definition 2.14. Let $\mathrm{Mr}$ be a category of rational maps from $X$ to torsors. Then $(u: X \rightarrow-u) \in \mathrm{Mr}$ is called a universal object for $\mathrm{Mr}$ if it admits the universal mapping property in Mr: For all $(\varphi: X \rightarrow P) \in \mathrm{Mr}$ there is a unique homomorphism of torsors $h: U \rightarrow P$ such that $\varphi=h \circ u$.

Remark 2.15. Universal objects are uniquely determined up to (unique) isomorphism.

Now assume that the base field $k$ is algebraically closed. (Arbitrary perfect base field is considered from Section 2.3.2 on.) In this case we may identify a torsor with the algebraic group acting on it (Remark 2.10), and a homomorphism of torsors becomes a homomorphism of algebraic groups composed with a translation (which is an isomorphism of torsors).

For the category Mav of morphisms from $X$ to abelian varieties (Definition 2.11) there exists a universal object, the Albanese mapping to the Albanese variety, denoted by alb : $X \rightarrow \operatorname{Alb}(X)$. This is a classical result; see [Lang 1959; Matsusaka 1952; Serre 1958-1959]. The Albanese variety $\operatorname{Alb}(X)$ is an abelian variety, dual to the Picard variety $\mathrm{Pic}_{X}^{0, \text { red }}$.

In the following we consider categories $\mathrm{Mr}$ of rational maps from $X$ to algebraic groups satisfying the following conditions:

$\left(1^{\diamond}\right) \mathrm{Mr}$ contains the category Mav.

$\left(2^{\diamond}\right)$ If $(\varphi: X \rightarrow G) \in \mathrm{Mr}$ and $h: G \rightarrow H$ is a homomorphism of torsors for smooth connected algebraic groups, then $h \circ \varphi \in$ Mr.

Theorem 2.16. Let $\mathrm{Mr}$ be a category of rational maps from $X$ to algebraic groups that satisfies $\left(1^{\diamond}\right)$ and $\left(2^{\diamond}\right)$ Then the following conditions are equivalent:

(i) For Mr there exists a universal object $(u: X \rightarrow U) \in M r$. 
(ii) There is a dual-algebraic formal subgroup $\mathscr{F}$ of $\underline{\mathrm{Div}}_{X}^{0, \text { red }}$ such that Mr is equivalent to $\operatorname{Mr}_{\mathscr{F}}(X)$. (iii) The formal group $\mathscr{F}_{\mathrm{Mr}} \subset \underline{\operatorname{Div}}_{X}^{0, \text { red }}$ generated by $\bigcup_{\varphi \in \mathrm{Mr}} \operatorname{im}\left(\tau_{\varphi}\right)$ is dual-algebraic
and $\operatorname{Mr}=\operatorname{Mr}_{\mathscr{F}_{\mathrm{Mr}}}(X)$.

Here $\operatorname{Mr}_{\mathscr{F}}(X)$ is the category of rational maps that induce a homomorphism of formal groups to $\mathscr{F}$ (Definition 2.13).

Proof. (ii) $\Longrightarrow$ (i) Assume that $\operatorname{Mr}$ is equivalent to $\operatorname{Mr}_{\mathscr{F}}(X)$, where $\mathscr{F}_{F}$ is a dualalgebraic formal group in $\underline{\operatorname{Div}}_{X}^{0, \text { red }}$. The first step is the construction of an algebraic

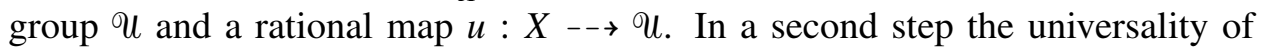
$u: X \rightarrow-\rightarrow$ for $\operatorname{Mr}_{\mathscr{F}}(X)$ will be shown.

Step 1: Construction of $u: X \rightarrow-\rightarrow$. $X$ is a smooth proper variety over $k$; thus the functor $\underline{\mathrm{Pic}}_{X}^{0}$ is represented by an algebraic group $\mathrm{Pic}_{X}^{0}$ whose underlying reduced scheme $\mathrm{Pic}_{X}^{0, \text { red }}$, the Picard variety of $X$, is an abelian variety. The class map $\underline{\text { Div }}_{X} \rightarrow \underline{\text { Pic }}_{X}$ induces a homomorphism $\mathscr{F} \rightarrow \mathrm{Pic}_{X}^{0, \text { red }}$.

We obtain a 1 -motive $M=\left[\mathscr{F} \rightarrow \mathrm{Pic}_{X}^{0, \text { red }}\right]$. Since $\mathrm{Pic}_{X}^{0, \text { red }}$ is an abelian variety, the dual 1-motive of $M$ is of the form $M^{\vee}=[0 \rightarrow G]$, where $G$ is a smooth connected algebraic group. Then define $\mathcal{U}$ to be this algebraic group. The canonical decomposition $0 \rightarrow \mathscr{L} \rightarrow \mathcal{U} \rightarrow \mathscr{A} \rightarrow 0$ is the extension of $\mathscr{A}=\operatorname{Alb}(X)=\left(\operatorname{Pic}_{X}^{0 \text {,red }}\right)^{\vee}$ by $\mathscr{L}=\mathscr{F}^{\vee}$ induced by the homomorphism $\mathscr{F} \rightarrow \mathrm{Pic}_{X}^{0, \text { red }}$ (Theorem 1.19).

Define the rational map $u: X \rightarrow-U_{\text {by }}$ the condition that the induced transformation $\tau_{u}: \mathscr{F} \rightarrow \underline{\operatorname{Div}}_{X}^{0 \text {,red }}$ from Definition 2.4 is the inclusion. According to Lemma 2.7, $u: X \rightarrow-\rightarrow$ is determined up to a constant $c \in \mathcal{U}(k)$.

Note that $u: X \rightarrow-\rightarrow$ generates $U$ : Let $H$ be the subgroup generated by the image of $u$, and let $\Lambda \subset \mathscr{L}$ be the affine part of $H$. Since $u: X \rightarrow \mathcal{U}$ factors through $H$, the induced transformation $\tau_{u}: \mathscr{L}^{\vee} \rightarrow \widehat{\mathrm{Div}_{X}}$ factors through the quotient $\mathscr{L}^{\vee} \rightarrow \Lambda^{\vee}$. Since $\tau_{u}$ is injective, this yields $\Lambda^{\vee} \cong \mathscr{L}^{\vee}$; hence $\Lambda \cong \mathscr{L}$. Since the composition $X \stackrel{u}{\stackrel{-}{\rightarrow}} \mathcal{U} \rightarrow \mathscr{A}$ generates $\mathscr{A}$, the abelian quotient of $H$ is $\mathscr{A}$. These two conditions imply that $H \cong \mathcal{U}$ by the five lemma.

Step 2: Universality of $u: X \rightarrow-\rightarrow$. Let $\varphi: X \rightarrow G$ be a rational map to a smooth connected algebraic group $G$ with canonical decomposition

$$
0 \rightarrow L \rightarrow G \stackrel{\rho}{\rightarrow} A \rightarrow 0
$$

inducing a homomorphism of formal groups $\tau_{\varphi}: L^{\vee} \rightarrow \mathscr{F} \subset \underline{\operatorname{Div}}_{X}^{0 \text {,red }}, \lambda \mapsto\left\langle\lambda, \ell_{\varphi}\right\rangle$ (Definition 2.4). Let $l:=\left(\tau_{\varphi}\right)^{\vee}: \mathscr{L} \rightarrow L$ be the dual homomorphism of affine groups. The composition

$$
X \stackrel{\varphi}{\rightarrow} G \stackrel{\rho}{\rightarrow} A
$$

extends to a morphism from $X$ to an abelian variety. Translating $\varphi$ by a constant $g \in G(k)$, if necessary, we may assume that $\rho \circ \varphi$ factors through $\mathscr{A}=\operatorname{Alb}(X)$. We 
are going to show that we have a commutative diagram

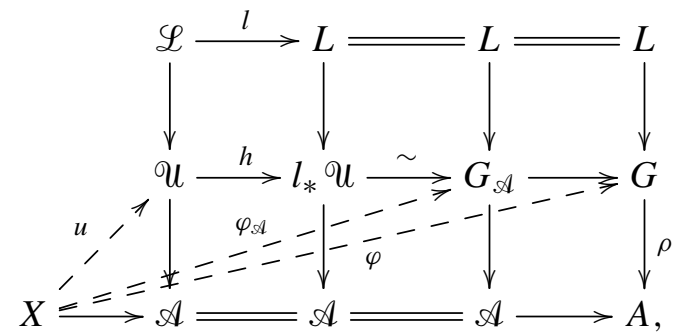

where $G_{\mathscr{A}}=G \times \times_{A} \mathscr{A}$ is the fiber product, $l_{*} \cup=U \amalg_{\mathscr{L}} L$ is the amalgamated sum and $h: \mathcal{U} \rightarrow l_{*} \cup$ is the map obtained from the amalgamated sum.

Denoting by $\bar{l}: \Gamma\left(\mathscr{L}\left(\mathscr{K}_{X}\right) / \mathscr{L}\left(\mathscr{O}_{X}\right)\right) \rightarrow \Gamma\left(L\left(\mathscr{K}_{X}\right) / L\left(\mathcal{O}_{X}\right)\right)$ the map induced by $l: \mathscr{L} \rightarrow L$, we have $\ell_{h \circ u}=\bar{l}\left(\ell_{u}\right)$. This yields

$$
\tau_{h \circ u}=\left\langle\cdot, \ell_{h \circ u}\right\rangle=\left\langle\cdot, \bar{l}\left(\ell_{u}\right)\right\rangle=\left\langle\cdot \circ l, \ell_{u}\right\rangle=\tau_{u} \circ l^{\vee}=\tau_{\varphi}
$$

since $\tau_{u}: \mathscr{F} \rightarrow \underline{\operatorname{Div}}_{X}^{0, \text { red }}$ is the inclusion by construction of $u$.

This implies that $l_{*} u_{X}$ and $G_{X}$ are isomorphic $L$-bundles over $X$. Then $l_{*} U$ and $G_{\mathscr{A}}$ are isomorphic as extensions of $\mathscr{A}$ by $L$, using the isomorphism $\operatorname{Pic}_{X}^{0} \stackrel{\sim}{\longrightarrow} \operatorname{Pic}_{\mathscr{A}}^{0}$. Thus $\tau_{h \circ u}=\tau_{\varphi}$ shows that $h \circ u$ and $\varphi_{\mathscr{A}}$ coincide up to translation. Since $u: X \rightarrow \mathcal{U}$ generates $\mathcal{U}$, each $h^{\prime}: \mathcal{U} \rightarrow G_{\mathscr{A}}$ fulfilling $h^{\prime} \circ u=\varphi_{\mathscr{A}}$ coincides with $h$. Hence $h$ is unique.

(i) $\Longrightarrow$ (iii) Assume $u: X \rightarrow \mathcal{U}$ is universal for Mr. Let $0 \rightarrow \mathscr{L} \rightarrow \mathcal{U} \rightarrow \mathscr{A} \rightarrow 0$ be the canonical decomposition of $U$, and let $\mathscr{F}$ be the image of the induced transformation $\tau_{u}: \mathscr{L}^{\vee} \rightarrow \underline{\operatorname{Div}}_{X}^{0, \text { red }}$. For $\lambda \in \mathscr{L}^{\vee}(R)$ the uniqueness of the homomorphism $h_{\lambda}: \cup \rightarrow \lambda_{*} \cup$ fulfilling $u_{\lambda}=h_{\lambda} \circ u$ implies that the rational maps $u_{\lambda}: X \rightarrow \lambda_{*} \cup$ are nonisomorphic to each other for distinct $\lambda \in \mathscr{L}^{\vee}(R)$. Hence $\operatorname{div}_{R}\left(u_{X, v}\right) \neq \operatorname{div}_{R}\left(u_{X, \lambda}\right)$ for $v \neq \lambda \in \mathscr{L}^{\vee}(R)$. Therefore $\mathscr{L}^{\vee} \rightarrow \mathscr{F}$ is injective and hence an isomorphism.

Let $\varphi: X \rightarrow G$ be an object of $\mathrm{Mr}$ and $0 \rightarrow L \rightarrow G \rightarrow A \rightarrow 0$ be the canonical decomposition of $G$. Translating $\varphi$ by a constant $g \in G(k)$, if necessary, we may assume that $\varphi: X \rightarrow G$ factors through a unique homomorphism $h: \mathcal{U} \rightarrow G$. The restriction of $h$ to $\mathscr{L}$ gives a homomorphism of affine groups $l: \mathscr{L} \rightarrow L$. Then the dual homomorphism $l^{\vee}: L^{\vee} \rightarrow \mathscr{F}_{F}$ yields a factorization of $L^{\vee} \rightarrow \underline{\operatorname{Div}}_{X}^{0, \text { red }}$ through $\mathscr{F}$. The properties $\left(1^{\diamond}\right),\left(2^{\diamond}\right)$ and the existence of a universal object guarantee that $\mathrm{Mr}$ contains all rational maps that induce a transformation to $\mathscr{F}$; hence $\mathrm{Mr}$ is equal to $\operatorname{Mr}_{\mathscr{F}}(X)$.

(iii) $\Longrightarrow$ (ii) is evident.

Notation 2.17. If $\mathscr{F}$ is a dual-algebraic formal subgroup of $\underline{\operatorname{Div}}_{X}^{0, \text { red }}$, then the universal object for $\operatorname{Mr}_{\mathscr{F}}(X)$ is denoted by $\operatorname{alb}_{\mathscr{F}}: X \rightarrow \operatorname{Alb}_{\mathscr{F}}(X)$. 
Remark 2.18. By construction, $\operatorname{Alb}_{\mathscr{F}}(X)$ is generated by $X$. Since $X$ is reduced, $\operatorname{Alb}_{\mathscr{F}}(X)$ is reduced as well and thus smooth. In the proof of Theorem 2.16 we have seen that $\operatorname{Alb}_{\mathscr{F}}(X)$ is an extension of the abelian variety $\operatorname{Alb}(X)$ by the affine group $\mathscr{F}^{\vee}$. More precisely, $\left[0 \rightarrow \operatorname{Alb}_{\mathscr{F}}(X)\right]$ is the dual 1 -motive of $\left[\mathscr{F}_{F} \rightarrow \mathrm{Pic}_{X}^{0, \text { red }}\right]$. The rational map $\left(\operatorname{alb}_{\mathscr{F}}: X \rightarrow \operatorname{Alb}_{\mathscr{F}}(X)\right) \in \operatorname{MrFF}_{\mathscr{F}}(X)$ is characterized by the fact that the transformation $\tau_{\mathrm{alb}_{\mathscr{F}}}: L^{\vee} \rightarrow \underline{\text { Div }}_{X}^{0, \text { red }}$ is the identity $\mathscr{F}_{\mathscr{F}} \stackrel{\text { id }}{\rightarrow} \mathscr{F}$.

2.3.2. Descent of the base field. Let $k$ be a perfect field. Let $\bar{k}$ be an algebraic closure of $k$. Let $X$ be a smooth proper variety defined over $k$, and write $\bar{X}=X \otimes_{k} \bar{k}$. Let $\mathscr{F}$ be a formal $k$-subgroup of $\underline{\operatorname{Div}}{ }_{X}^{0}$, and write $\overline{\mathscr{F}}=\mathscr{F} \widehat{\otimes} \bar{k}$.

The wish is to show that the universal object $\operatorname{alb}_{\overline{\mathscr{F}}}: \bar{X} \rightarrow \operatorname{Alb}_{\overline{\mathscr{F}}}(\bar{X})$ for the category $\mathrm{Mr}_{\bar{F}}$ can be defined over $k$. This will be accomplished by a Galois descent, as described in [Serre 1959, V, §4]. Due to Cartier duality between formal groups and affine groups (Theorem 1.5), Galois descent applies to formal groups as well.

When one does not assume that $X$ is endowed with a $k$-rational point, one is led to two different descents of $\operatorname{Alb}_{\bar{F}}(\bar{X})$ :

First: The universal mapping property of $\operatorname{alb}_{\overline{\mathscr{F}}}: \bar{X} \rightarrow \operatorname{Alb}_{\overline{\mathscr{F}}}(\bar{X})$ gives for every $\sigma \in \operatorname{Gal}(\bar{k} / k)$ transformations $h_{\sigma}^{(1)}: \operatorname{Alb}_{\overline{\mathscr{F}}}(\bar{X}) \rightarrow \operatorname{Alb}_{\overline{\mathscr{F}}}(\bar{X})^{\sigma}$ between $\operatorname{Alb}_{\overline{\mathscr{F}}}(\bar{X})$ and its conjugate $\operatorname{Alb}_{\overline{\mathscr{F}}}(\bar{X})^{\sigma}$, which are homomorphisms of torsors. Therefore the descent of $\operatorname{Alb}_{\bar{F}}(\bar{X})$ by means of the $h_{\sigma}^{(1)}$ yields a $k$-torsor $\operatorname{Alb}_{\mathscr{F}}^{(1)}(X)$.

Second: To avoid translations or the reference to base points, one may reformulate the universal mapping property, replacing rational maps $\varphi: \bar{X} \rightarrow G$ from $\bar{X}$ to algebraic groups by its associated "difference maps" $\varphi^{(-)}: \bar{X} \times \bar{X} \rightarrow G$, $(p, q) \mapsto \varphi(q)-\varphi(p)$. In this way translations are eliminated and one obtains transformations $h_{\sigma}^{(0)}: \operatorname{Alb}_{\bar{F}}(\bar{X}) \rightarrow \operatorname{Alb}_{\overline{\mathscr{F}}}(\bar{X})^{\sigma}$ that are homomorphisms of algebraic groups. Then the descent of $\operatorname{Alb}_{\overline{\mathscr{F}}}(\bar{X})$ by means of the $h_{\sigma}^{(0)}$ yields an algebraic $k$-group $\operatorname{Alb}_{\mathscr{F}}^{(0)}(X)$. This is the $k$-group acting on the $k$-torsor $\operatorname{Alb}_{\mathscr{F}}^{(1)}(X)$.

Notation 2.19. If $\varphi: X \rightarrow P$ is a rational map to a torsor $P$ for an algebraic group $G$, then $\varphi^{(-)}: X \times X \rightarrow G$ denotes the rational map to the algebraic group $G$ that assigns for $S \in \operatorname{Alg} / k$ to $(p, q) \in X(S) \times X(S)$ the unique $g \in G(S)$ such that $g \cdot \varphi(p)=\varphi(q)$.

Notation 2.20. If $\varphi: X \rightarrow P$ is a rational map to a torsor, then set $\varphi^{(1)}:=\varphi$, $\varphi^{(0)}:=\varphi^{(-)}$.

Theorem 2.21. There exists a $k$-torsor $\operatorname{Alb}_{\mathscr{F}}^{(1)}(X)$ for an algebraic k-group $\operatorname{Alb}_{\mathscr{F}}^{(0)}(X)$ and rational maps defined over $k$

$$
\operatorname{alb}_{\mathscr{F}}^{(i)}: X^{2-i} \rightarrow \operatorname{Alb}_{\mathscr{F}}^{(i)}(X) \quad \text { for } i=1,0
$$

satisfying the following universal property: 
If $\varphi: X \rightarrow G^{(1)}$ is a rational map defined over $k$ to a $k$-torsor $G^{(1)}$ for an algebraic $k$-group $G^{(0)}$ that is an object of $\operatorname{MrFr}_{\mathscr{F}}(X)$, then there exist unique homomorphisms of $k$-torsors and algebraic $k$-groups

$$
h^{(1)}: \operatorname{Alb}_{\mathscr{F}}^{(1)}(X) \rightarrow G^{(1)} \quad \text { and } \quad h^{(0)}: \operatorname{Alb}_{\mathscr{F}}^{(0)}(X) \rightarrow G^{(0)},
$$

respectively, defined over $k$, such that $\varphi^{(i)}=h^{(i)} \circ \operatorname{alb}_{\mathscr{F}}^{(i)}$ for $i=1,0$.

The algebraic group $\operatorname{Alb}_{\mathscr{F}}^{(0)}(X)$ is dual to the 1-motive $\left[\mathscr{F}^{\rightarrow} \mathrm{Pic}_{X}^{0, \text { red }}\right]$.

Proof. Galois descent. The same arguments as given in [Serre 1959, V, no. 22] work in our situation.

2.3.3. Functoriality. Let $\mathscr{F} \subset \underline{\operatorname{Div}}_{X}^{0, \text { red }}$ be a dual-algebraic formal $k$-group. Let $\psi: Y \rightarrow X$ be a $k$-morphism of smooth proper $k$-varieties, such that no irreducible component of $\psi(Y)$ is contained in $\operatorname{Supp}(\mathscr{F})$. For each dual-algebraic formal $k$-group $\varphi \subset \operatorname{Div}_{Y}^{0, \text { red }}$ containing $\psi^{*} \mathscr{F}_{F}$ the pull-back of relative Cartier divisors and of line bundles induces a homomorphism $\left[\mathscr{G} \rightarrow \mathrm{Pic}_{Y}^{0, \text { red }}\right] \leftarrow\left[\mathscr{F}_{F} \rightarrow \mathrm{Pic}_{X}^{0 \text {,red }}\right]$ of 1-motives.

According to the construction of universal objects over $\bar{k}$ (Remark 2.18), we obtain via dualization of 1-motives and by Galois descent:

Proposition 2.22. Using the assumptions above, $\psi$ induces for every formal group $\varphi \subset \underline{\operatorname{Div}}_{Y}^{0, \text { red }}$ containing $\psi^{*} \mathscr{F}_{F}$ a homomorphism of $k$-torsors $\operatorname{Alb}_{(\mathcal{G}}^{(1)}(\psi)$ and $a$ homomorphism of algebraic k-groups $\operatorname{Alb}^{(0) \mathscr{F}}(\psi)$,

$$
\operatorname{Alb}^{(i)} \underset{\mathscr{F}}{\mathscr{F}}(\psi): \operatorname{Alb}_{\mathscr{G}}^{(i)}(Y) \rightarrow \operatorname{Alb}_{\mathscr{F}}^{(i)}(X) \quad \text { for } i=1,0 .
$$

\section{Albanese with modulus}

Let $X$ be a smooth proper variety over a perfect field $k$. Let $D$ be an effective divisor on $X$ (with multiplicity). The Albanese $\operatorname{Alb}^{(1)}(X, D)$ of $X$ of modulus $D$ is a higher-dimensional analogue of the generalized Jacobian with modulus of Rosenlicht and Serre. $\operatorname{Alb}^{(1)}(X, D)$ is defined by the universal mapping property for morphisms from $X \backslash D$ to torsors of modulus $\leq D$ (Definition 3.11). Our definition of the modulus of rational maps coincides with the classical definition from [Serre 1959, III, §1] in the curve case. Therefore the Albanese with modulus agrees with the Jacobian with modulus of Rosenlicht and Serre for curves, which we review in Section 3.3.

In Section 3.4 we consider a Chow group $\mathrm{CH}_{0}(X, D)^{0}$ of 0 -cycles relative to the modulus $D$ (Definition 3.27). We give an alternative characterization of $\operatorname{Alb}(X, D)$ as a universal quotient of $\mathrm{CH}_{0}(X, D)^{0}$, when the base field is algebraically closed (Theorem 3.29). 
3.1. Filtrations of the Witt group. Here we present a global version of some basic notions from [Kato and Russell 2010] that are needed for the construction of the Albanese with modulus.

Let $(K, \mathrm{v})$ be a discrete valuation field of characteristic $p>0$ with residue field $k$. The group of Witt vectors of length $r$ is denoted by $W_{r}$ (Example 1.11).

Definition 3.1. Let fil $_{n} W_{r}(K)$ be the subgroup of $W_{r}(K)$ from [Brylinski 1983, Section 1, Proposition 1]:

$$
\mathrm{fil}_{n} W_{r}(K)=\left\{\left(f_{r-1}, \ldots, f_{0}\right) \mid f_{i} \in K, \mathrm{v}\left(f_{i}\right) \geq-n / p^{i} \text { for all } 0 \leq i \leq r-1\right\} .
$$

Let $\mathrm{fil}_{n}^{\mathrm{F}} W_{r}(K)$ be the subgroup of $W_{r}(K)$ generated by $\mathrm{fil}_{n} W_{r}(K)$ by means of the Frobenius F (see [Kato and Russell 2010, 2.2]):

$$
\mathrm{fil}_{n}^{\mathrm{F}} W_{r}(K)=\sum_{\nu \geq 0} \mathrm{~F}^{v} \mathrm{fil}_{n} W_{r}(K) .
$$

Let $X$ be a variety over $k$, regular in codimension 1 . Let $D=\sum_{q \in S} n_{q} D_{q}$ be an effective divisor on $X$, where $S$ is a finite set of points of codimension 1 in $X$, where $D_{q}$ are the prime divisors associated to $q \in S$ and $n_{q}$ are positive integers for $q \in S$.

Definition 3.2. Let $\mathrm{fil}_{D} W_{r}\left(\mathscr{K}_{X}\right)$ and $\mathrm{fil}_{D}^{\mathrm{F}} W_{r}\left(\mathscr{K}_{X}\right)$ be the sheaves of subgroups of $W_{r}\left(\mathscr{K}_{X}\right)$ formed by the groups

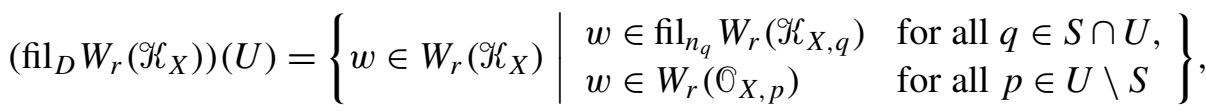

$$
\begin{aligned}
& \left(\operatorname{fil}_{D}^{\mathrm{F}} W_{r}\left(\mathscr{K}_{X}\right)\right)(U)=\left\{\begin{array}{l|l}
w \in W_{r}\left(\mathscr{K}_{X}\right) & \begin{array}{ll}
w \in \mathrm{fil}_{n_{q}}^{\mathrm{F}} W_{r}\left(\mathscr{K}_{X, q}\right) & \text { for all } q \in S \cap U, \\
w \in W_{r}\left(\mathcal{O}_{X, p}\right) & \text { for all } p \in U \backslash S
\end{array}
\end{array}\right\} \text {, }
\end{aligned}
$$

respectively, for open $U \in X$, where $\mathrm{fil}_{n} W_{r}\left(\mathscr{K}_{X, q}\right)$ and $\mathrm{fil}_{n}^{\mathrm{F}} W_{r}\left(\mathscr{K}_{X, q}\right)$ denote the filtrations associated to the valuation $\mathrm{v}_{q}$ attached to the point $q \in S$.

Proposition 3.3. Suppose $X$ is a projective variety over $k$ and $D$ an effective divisor on $X$. Then $\Gamma\left(X, \mathrm{fil}_{D} W_{r}\left(\mathscr{K}_{X}\right)\right)$ is a finite $W_{r}(k)$-module.

Proof. The Verschiebung V: $W_{r-1} \rightarrow W_{r}$ yields an exact sequence

$$
0 \rightarrow \operatorname{fil}_{D} W_{r-1}\left(\mathscr{K}_{X}\right) \rightarrow \mathrm{fil}_{D} W_{r}\left(\mathscr{K}_{X}\right) \rightarrow \mathrm{fil}_{\left\lfloor D / p^{r-1}\right\rfloor} W_{1}\left(\mathscr{K}_{X}\right) \rightarrow 0,
$$

where $\left\lfloor D / p^{r-1}\right\rfloor=\sum_{q \in S}\left\lfloor n_{q} / p^{r-1}\right\rfloor D_{q}$. This induces the exact sequence

$$
0 \rightarrow \Gamma\left(\mathrm{fil}_{D} W_{r-1}\left(\mathscr{K}_{X}\right)\right) \rightarrow \Gamma\left(\mathrm{fil}_{D} W_{r}\left(\mathscr{K}_{X}\right)\right) \rightarrow \Gamma\left(\mathrm{fil}_{\left\lfloor D / p^{r-1}\right\rfloor} W_{1}\left(\mathscr{K}_{X}\right)\right) .
$$

By induction over $r \geq 1$ and since $W_{1}(k)=k$ is noetherian, it is sufficient to show the statement for $r=1$. Now $\operatorname{fil}_{D} W_{1}\left(\mathscr{K}_{X}\right)=\mathrm{O}_{X}(D)$ is a coherent sheaf, and hence $\Gamma\left(X, \mathrm{fil}_{D} W_{1}\left(\mathscr{K}_{X}\right)\right)$ is a finite module over $W_{1}(k)=k$. 
Definition 3.4. Let $R$ be a commutative ring over $\mathbb{F}_{p}$. We let $R[\mathrm{~F}]$ be the noncommutative polynomial ring defined by

$$
R[\mathrm{~F}]=\left\{\sum_{i=1}^{n} \mathrm{~F}^{i} r_{i} \mid r_{i} \in R, n \in \mathbb{N}\right\}, \quad \text { where } \mathrm{F} r=r^{p} \mathrm{~F} \quad \text { for all } r \in R .
$$

Definition 3.5. If $\Omega_{\mathscr{K}_{X}}=\Omega_{\mathscr{K}_{X} / k}$ is the module of differentials of $\mathscr{K}_{X}$ over $k$, we let $\delta$ be the homomorphism

$$
\delta: W_{r}\left(\mathscr{K}_{X}\right) \rightarrow \Omega_{\mathscr{K}_{X}}, \quad\left(f_{r-1}, \ldots, f_{0}\right) \mapsto \sum_{i=0}^{r-1} f_{i}^{p^{i}-1} \mathrm{~d} f_{i} .
$$

Definition 3.6. If $E$ is a reduced effective divisor on $X$ with normal crossings, we let $\Omega_{X}(\log E)$ be the sheaf of differentials on $X$ with $\log$-poles along $E$, that is, the $O_{X}$-module generated locally by $\mathrm{d} f$ for $f \in \mathcal{O}_{X}$ and $\mathrm{d} \log t=t^{-1} \mathrm{~d} t$, where $t$ is a local equation for $E$.

Proposition 3.7. Suppose $D_{\mathrm{red}}$ is a normal crossing divisor. The homomorphism $\delta$ from Definition 3.5 induces injective homomorphisms

$$
\begin{aligned}
& \mathfrak{d}_{D}: \operatorname{fil}_{D}^{\mathrm{F}} W_{r}\left(\mathscr{K}_{X}\right) / \operatorname{fil}_{\lfloor D / p\rfloor}^{\mathrm{F}} W_{r}\left(\mathscr{K}_{X}\right) \rightarrow \mathfrak{D}_{D}, \\
& \overline{\mathfrak{d}}_{D}: \operatorname{fil}_{D}^{\mathrm{F}} W_{r}\left(\mathscr{K}_{X}\right) / \operatorname{fil}_{D-D_{\text {red }}}^{\mathrm{F}} W_{r}\left(\mathscr{K}_{X}\right) \rightarrow \overline{\mathfrak{D}}_{D},
\end{aligned}
$$

where $\mathfrak{D}_{D}$ and $\overline{\mathfrak{D}}_{D}$ are the $\mathfrak{O}_{X}$-modules

$$
\begin{aligned}
& \mathfrak{D}_{D}=k[\mathrm{~F}] \otimes_{k}\left(\Omega_{X}\left(\log D_{\text {red }}\right) \otimes_{\mathcal{O}_{X}} \mathscr{O}_{X}(D) / \mathscr{O}_{X}(\lfloor D / p\rfloor)\right), \\
& \overline{\mathfrak{D}}_{D}=k[\mathrm{~F}] \otimes_{k}\left(\Omega_{X}\left(\log D_{\text {red }}\right) \otimes_{\mathcal{O}_{X}} \mathscr{O}_{X}(D) / \mathscr{O}_{X}\left(D-D_{\text {red }}\right)\right),
\end{aligned}
$$

and $\lfloor D / p\rfloor$ means the largest divisor $E$ such that $p E \leq D$.

Proof. This is the global formulation of [Kato and Russell 2010, 4.6].

Definition 3.8. Let ${ }^{b} \overline{\mathfrak{D}}_{D}$ be the image in $\overline{\mathfrak{D}}_{D}$ of the $\mathrm{O}_{X}$-module

$$
k[\mathrm{~F}] \otimes_{k}\left(\Omega_{X} \otimes_{\mathrm{O}_{X}} \mathrm{O}_{X}(D) / \mathscr{O}_{X}\left(D-D_{\mathrm{red}}\right)\right)
$$

(without log-poles). Then

$$
{ }^{b} \overline{\mathfrak{D}}_{D} \cong k[\mathrm{~F}] \otimes_{k}\left(\Omega_{D_{\text {red }}} \otimes_{\mathscr{O}_{D_{\text {red }}}} \mathcal{O}_{X}(D) / \mathcal{O}_{X}\left(D-D_{\text {red }}\right)\right)
$$

since $t^{-n_{q}} \mathrm{~d} t=t^{1-n_{q}} \mathrm{~d} \log t$ vanishes in $\overline{\mathfrak{D}}_{D}$ for any local equation $t$ of $D_{\text {red }}$. Then we let ${ }^{\mathrm{b}} \mathrm{fil}_{D}^{\mathrm{F}} W_{r}\left(\mathscr{K}_{X}\right) \subset \mathrm{fil}_{D}^{\mathrm{F}} W_{r}\left(\mathscr{K}_{X}\right)$ be the inverse image of ${ }^{b} \overline{\mathfrak{D}}_{D}$ under the map $\overline{\mathfrak{d}}_{D}$ from Proposition 3.7. According to [Kato and Russell 2010, 4.7], this is a global version of the following alternative definition: 
Definition 3.9. Let ${ }^{b} \mathrm{fil}_{n} W_{r}(K)$ be the subgroup of $\mathrm{fil}_{n} W_{r}(K)$ consisting of all elements $\left(f_{r-1}, \ldots, f_{0}\right)$ satisfying the following condition: If the $p$-adic order $v$ of $n$ is less than $r$, then $p^{v} \mathrm{v}\left(f_{v}\right)>-n$. Then ${ }^{\mathrm{b}} \mathrm{fil}_{n}^{\mathrm{F}} W_{r}(K)$ is the subgroup of $W_{r}(K)$ generated by ${ }^{\mathrm{b}} \mathrm{fil}_{n} W_{r}(K)$ by means of the Frobenius $\mathrm{F}$,

$$
{ }^{\mathrm{b}} \mathrm{fil}_{n}^{\mathrm{F}} W_{r}(K)=\sum_{\nu \geq 0} \mathrm{~F}^{v \mathrm{~b}} \mathrm{fil}_{n} W_{r}(K) .
$$

Lemma 3.10. Let $\psi: Y \rightarrow X$ be a morphism of varieties over $k$, such that $\psi(Y)$ intersects $\operatorname{Supp}(D)$ properly. Let $D \cdot Y$ denote the pull-back of $D$ to $Y$. Suppose that $D_{\mathrm{red}}$ and $(D \cdot Y)_{\mathrm{red}}$ are normal crossing divisors. There is a commutative diagram of homomorphisms with injective rows

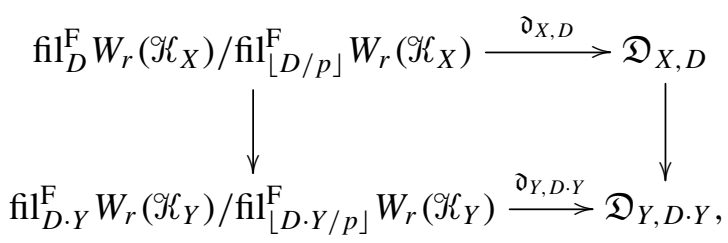

where the vertical arrows are the obvious pull-back maps from $X$ to $Y$.

Proof. Straightforward.

\subsection{Albanese with modulus.}

3.2.1. Existence and construction. Let $X$ be a smooth proper variety over a perfect field $k$.

Definition 3.11. First assume $k$ is algebraically closed. Let $\varphi: X \rightarrow G$ be a rational map from $X$ to a smooth connected algebraic group $G$. Let $L$ be the affine part of $G$ and $U$ the unipotent part of $L$. The modulus of $\varphi$ from [Kato and Russell $2010, \S 3]$ is the following effective divisor

$$
\bmod (\varphi)=\sum_{\operatorname{ht}(q)=1} \bmod _{q}(\varphi) D_{q}
$$

where $q$ ranges over all points of codimension 1 in $X$, and $D_{q}$ is the prime divisor associated to $q$. For each $q \in X$ of codimension 1, the canonical map $L\left(\mathscr{K}_{X, q}\right) / L\left(\mathcal{O}_{X, q}\right) \rightarrow G\left(\mathscr{K}_{X, q}\right) / G\left(\mathcal{O}_{X, q}\right)$ is bijective; see Section 2.2.1. Take an element $l_{q} \in L\left(\mathscr{K}_{X, q}\right)$ whose image in $G\left(\mathscr{K}_{X, q}\right) / G\left(\mathscr{O}_{X, q}\right)$ coincides with the class of $\varphi \in G\left(\mathscr{K}_{X, q}\right)$. If $\operatorname{char}(k)=0$, let $\left(u_{q, i}\right)_{1 \leq i \leq s}$ be the image of $l_{q}$ in $\mathbb{G}_{\mathrm{a}}\left(\mathscr{K}_{X, q}\right)^{s}$ under $L \rightarrow U \cong\left(\mathbb{G}_{\mathrm{a}}\right)^{s}$. If $\operatorname{char}(k)=p>0$, let $\left(u_{q, i}\right)_{1 \leq i \leq s}$ be the image of $l_{q}$ in $W_{r}\left(\mathscr{K}_{X, q}\right)^{s}$ under $L \rightarrow U \subset\left(W_{r}\right)^{s}$.

$$
\bmod _{q}(\varphi)= \begin{cases}0 & \text { if } \varphi \in G\left(\mathcal{O}_{X, q}\right), \\ 1+\max \left\{\mathrm{n}_{q}\left(u_{q, i}\right) \mid 1 \leq i \leq s\right\} & \text { if } \varphi \notin G\left(\mathcal{O}_{X, q}\right),\end{cases}
$$


where for $u \in \mathbb{G}_{\mathrm{a}}\left(\mathscr{K}_{X, q}\right)$ if $\operatorname{char}(k)=0$, or $W_{r}\left(\mathscr{K}_{X, q}\right)$ if $\operatorname{char}(k)=p>0$, we denote

$$
\mathrm{n}_{q}(u)= \begin{cases}-\mathrm{v}_{q}(u) & \text { if } \operatorname{char}(k)=0, \\ \min \left\{n \in \mathbb{N} \mid u \in \operatorname{fil}_{n}^{\mathrm{F}} W_{r}\left(\mathscr{K}_{X, q}\right)\right\} & \text { if } \operatorname{char}(k)=p>0 .\end{cases}
$$

Note that $\bmod _{v}(\varphi)$ is independent of the choice of the isomorphism $U \cong\left(\mathbb{G}_{\mathrm{a}}\right)^{s}$ or, respectively, of the embedding $U \subset\left(W_{r}\right)^{s}$; see [Kato and Russell 2010, Theorem 3.3].

For arbitrary perfect base field $k$ and $G$ a torsor for a smooth connected algebraic group we obtain $\bmod (\varphi)$ by means of a Galois descent from $\bmod \left(\varphi \otimes_{k} \bar{k}\right)$, where $\bar{k}$ is an algebraic closure of $k$; see [Kato and Russell 2010, No. 3.4] and Remark 2.10.

Definition 3.12. Let $D$ be an effective divisor on $X$. Then $\operatorname{Mr}(X, D)$ denotes the category of those rational maps $\varphi$ from $X$ to torsors such that $\bmod (\varphi) \leq D$. The universal object of $\operatorname{Mr}(X, D)$ (if it exists) is called the Albanese of $X$ of modulus $D$ and denoted by $\operatorname{Alb}^{(1)}(X, D)$, or just $\operatorname{Alb}(X, D)$, if it admits a $k$-rational point (cf. Remark 2.10).

Remark 3.13. In the definition of $\bmod (\varphi)$ (Definition 3.11) we used, instead of the original filtration fil. $W$ of Brylinski, the saturation fil ${ }_{\bullet}^{\mathrm{F}} W$ of fil. $W$ with respect to the Frobenius. This is motivated as follows: $\operatorname{Let}^{\bmod ^{\exists}}(\varphi)$ be the modulus of a rational map $\varphi$ using the filtration fil. $W$ instead of fil. $W$. If $\varphi: X \rightarrow \mathbb{G}_{\mathrm{a}}$ is a nonconstant rational map, that is, the multiplicity of $\bmod (\varphi)=: D$ is greater than 1 , then $\bmod ^{\exists}\left(\mathrm{F}^{v} \circ \varphi\right)=p^{v}\left(D-D_{\text {red }}\right)+D_{\text {red }}$, where $D_{\text {red }}$ is the reduced part of $D$. On the other hand, if $u: X \rightarrow U$ is a universal object for a certain category of rational maps $\mathrm{Mr}$, then clearly $u$ satisfies the universal mapping property as well for all maps of the form $\mathrm{F}^{v} \circ \varphi, \varphi \in \operatorname{Mr}$ (cf. condition ( $2^{\diamond}$ ) before Theorem 2.16). This shows that $\bmod ^{\exists}(\varphi)$ is not compatible with the notion of universal objects.

Definition 3.14. Let $D$ be an effective divisor on $X$, and let $D_{\text {red }}$ be the reduced part of $D$. Then $\mathscr{F}_{X, D}$ denotes the formal subgroup of $\underline{\operatorname{Div}}_{X}$ characterized by

$$
\left(\mathscr{F}_{X, D}\right)_{\text {ét }}=\left\{B \in \underline{\operatorname{Div}}_{X}(k) \mid \operatorname{Supp}(B) \subset \operatorname{Supp}(D)\right\},
$$

and for $\operatorname{char}(k)=0$,

$$
\left(\mathscr{F}_{X, D}\right)_{\text {inf }}=\exp \left(\widehat{\mathbb{G}}_{\mathrm{a}} \otimes_{k} \Gamma\left(\mathscr{O}_{X}\left(D-D_{\text {red }}\right) / \mathscr{O}_{X}\right)\right),
$$

for $\operatorname{char}(k)=p>0$,

$$
\left(\mathscr{F}_{X, D}\right)_{\text {inf }}=\operatorname{Exp}\left(\sum_{r>0}{ }_{r} \widehat{W} \otimes_{W_{r}(k)} \Gamma\left(\mathrm{fil}_{D-D_{\text {red }}}^{\mathrm{F}} W_{r}\left(\mathscr{K}_{X}\right) / W_{r}\left(\mathbb{O}_{X}\right)\right), 1\right) .
$$

Let $\mathscr{F}_{X, D}^{0, \text { red }}=\mathscr{F}_{X, D} \times_{\underline{\operatorname{Div}}_{X}} \underline{\operatorname{Div}}_{X}^{0, \text { red }}$ be the intersection of $\mathscr{F}_{X, D}$ and $\underline{\operatorname{Div}}_{X}^{0, \text { red }}$.

Proposition 3.15. The formal groups $\mathscr{F}_{X, D}$ and $\mathscr{F}_{X, D}^{0, \text { red }}$ are dual-algebraic. 
Proof. The statement is obvious for $\operatorname{char}(k)=0$, so we suppose $\operatorname{char}(k)=p>0$. The proof is done in two steps. Let $\mathscr{F}_{X, D}^{\dagger}$ be the formal subgroup of $\underline{\operatorname{Div}}_{X}$ defined in the same way as $\mathscr{F}_{X, D}$, but using the filtration fil $_{D-D_{\text {red }}} W_{r}\left(\mathscr{K}_{X}\right)$ instead of $\mathrm{fil}_{D-D_{\text {red }}}^{\mathrm{F}} W_{r}\left(\mathscr{K}_{X}\right)$. In the first step, we show that for any effective divisor $D$ the formal group $\mathscr{F}_{X, D}^{ \pm}$is dual-algebraic. In the second step, we show that for any $D$ there exists $D^{\prime} \geq D$ such that $\mathscr{F}_{X, D}$ is contained in the image of $\mathscr{F}_{X, D^{\prime}}^{\dagger}$ in $\mathscr{F}_{X, D^{\prime}}$. Thus $\mathscr{F}_{X, D}$ is a formal subgroup of a quotient of a dual-algebraic formal group, and hence dual-algebraic by Lemma 1.17 . Then also the formal subgroup $\mathscr{F}_{X, D}^{0 \text {,red }}$ of $\mathscr{F}_{X, D}$ is dual-algebraic.

Step 1. Let $D$ be an effective divisor on $X$. Write $D=\sum_{\mathrm{ht}(q)=1} n_{q} D_{q}$, where $q$ ranges over all points of codimension 1 in $X$, and $D_{q}$ is the prime divisor associated to $q$. Let $S$ be the finite set of those $q$ with $n_{q}>0$. Let

$$
m=\min \left\{r \mid p^{r}>n_{q}-1 \text { for all } q \in S\right\} .
$$

Hence for $r \geq m$, if $\left(f_{r-1}, \ldots, f_{0}\right) \in$ fil $_{D-D_{\text {red }}} W_{r}\left(\mathscr{K}_{X}\right)$, then $f_{i} \in \mathcal{O}_{X}$ for $r>i \geq m$, according to Definition 3.2. Then the Verschiebung $\mathrm{V}^{r-m}: W_{m}\left(\mathscr{K}_{X}\right) \rightarrow W_{r}\left(\mathscr{K}_{X}\right)$ yields a surjective homomorphism

$$
\mathrm{fil}_{D-D_{\text {red }}} W_{m}\left(\mathscr{K}_{X}\right) / W_{m}\left(\mathcal{O}_{X}\right) \rightarrow \mathrm{fil}_{D-D_{\text {red }}} W_{r}\left(\mathscr{K}_{X}\right) / W_{r}\left(\mathcal{O}_{X}\right) .
$$

Thus $\left(\mathscr{F}_{X, D}^{\exists}\right)_{\text {inf }}$ is already generated by a finite sum via Exp:

$$
\left(\mathscr{F}_{X, D}^{\dagger}\right)_{\text {inf }}=\operatorname{Exp}\left(\sum_{1 \leq r \leq m}{ }_{r} \widehat{W} \otimes_{W_{r}(k)} \Gamma\left(\operatorname{fil}_{D-D_{\text {red }}} W_{r}\left(\mathscr{K}_{X}\right) / W_{r}\left(\mathcal{O}_{X}\right)\right), 1\right) .
$$

Each $\Gamma\left(X\right.$, fil $\left._{D-D_{\text {red }}} W_{r}\left(\mathscr{K}_{X}\right) / W_{r}\left(\mathcal{O}_{X}\right)\right)$ is a finitely generated $W_{r}(k)$-module, by the same proof as for Proposition 3.3. Hence $\left(\mathscr{F}_{X, D}^{t}\right)_{\text {inf }}$ is a quotient of the direct sum of finitely many ${ }_{r} \widehat{W}$.

Moreover, $\left.\left(\mathscr{F}_{X, D}^{\dagger}\right)\right)_{\text {ét }}=\left(\mathscr{F}_{X, D}\right)_{\text {ét }}$ is an abelian group of finite type, since $D$ has only finitely many components. Thus $\mathscr{F}_{X, D}^{\lrcorner}$is dual-algebraic, according to Proposition 1.16.

Step 2. We show that for any effective divisor $D$ there exists an effective divisor $D^{\prime} \geq D$ such that $\mathscr{F}_{X, D}$ is generated by $\mathscr{F}_{X, D^{\prime}}^{\lrcorner}$. We will find an effective divisor $D^{\prime} \geq D$ such that $\Gamma\left(\mathrm{fil}_{D-D_{\text {red }}}^{\mathrm{F}} W_{r}\left(\mathscr{K}_{X}\right) / W_{r}\left(\mathcal{O}_{X}\right)\right)$ is generated by

$$
\sum_{\nu \geq 0} \mathrm{~F}^{\nu} \Gamma\left(\mathrm{fil}_{D^{\prime}-D_{\mathrm{red}}^{\prime}} W_{r}\left(\mathscr{K}_{X}\right) / W_{r}\left(\mathcal{O}_{X}\right)\right) .
$$

This is sufficient because

$$
\operatorname{Exp}\left(v \otimes \sum_{i} \mathrm{~F}^{v_{i}} \omega_{i}, 1\right)=\operatorname{Exp}\left(\sum_{i} \mathrm{~V}^{v_{i}} v \otimes \omega_{i}, 1\right) .
$$


Since the homomorphism

$$
\mathrm{V}^{r-m}: \operatorname{fil}_{D-D_{\text {red }}}^{\mathrm{F}} W_{m}\left(\mathscr{K}_{X}\right) / W_{m}\left(\mathcal{O}_{X}\right) \rightarrow \mathrm{fil}_{D-D_{\text {red }}}^{\mathrm{F}} W_{r}\left(\mathscr{K}_{X}\right) / W_{r}\left(\mathcal{O}_{X}\right)
$$

is surjective for $r \geq m$, we only need to consider $r=m$.

The exact sequence

$$
0 \rightarrow W_{r}\left(\mathscr{O}_{X}\right) \rightarrow W_{r}\left(\mathscr{K}_{X}\right) \rightarrow W_{r}\left(\mathscr{K}_{X}\right) / W_{r}\left(\mathscr{O}_{X}\right) \rightarrow 0
$$

yields the exact sequence

$$
\Gamma\left(W_{r}\left(\mathscr{K}_{X}\right)\right) \rightarrow \Gamma\left(W_{r}\left(\mathscr{K}_{X}\right) / W_{r}\left(\mathrm{O}_{X}\right)\right) \rightarrow \mathrm{H}^{1}\left(W_{r}\left(\mathcal{O}_{X}\right)\right) \rightarrow 0 .
$$

Here $\mathrm{H}^{1}\left(W_{r}\left(\mathscr{K}_{X}\right)\right)=0$ since $W_{r}\left(\mathscr{K}_{X}\right)$ is a flasque sheaf. Since $\mathrm{H}^{1}\left(W_{r}\left(\mathrm{O}_{X}\right)\right)$ is a finite $W_{r}(k)$-module, there is an effective divisor $E$ such that the map

$$
\Gamma\left(\mathrm{fil}_{E} W_{r}\left(\mathscr{K}_{X}\right) / W_{r}\left(\mathrm{O}_{X}\right)\right) \rightarrow \mathrm{H}^{1}\left(W_{r}\left(\mathrm{O}_{X}\right)\right)
$$

is surjective. Hence for any $\sigma \in \Gamma\left(\mathrm{fil}_{D-D_{\text {red }}}^{\mathrm{F}} W_{r}\left(\mathscr{K}_{X}\right) / W_{r}\left(\mathscr{O}_{X}\right)\right)$ there is

$$
\rho \in \Gamma\left(\mathrm{fil}_{E} W_{r}\left(\mathscr{K}_{X}\right) / W_{r}\left(\mathscr{O}_{X}\right)\right)
$$

such that $\sigma-\rho$ lies in the image of $\Gamma\left(W_{r}\left(\mathscr{K}_{X}\right)\right)$, and hence in the image of $\Gamma\left(\right.$ fil $\left._{E^{\prime}}^{\mathrm{F}} W_{r}\left(\mathscr{K}_{X}\right)\right)$, where $E^{\prime}=\max \left\{E, D-D_{\text {red }}\right\}$. Therefore we are reduced to showing that for any $D$ there exists $D^{\prime} \geq D$ such that $\Gamma\left(\mathrm{fil}_{D}^{\mathrm{F}} W_{r}\left(\mathscr{K}_{X}\right)\right)$ is generated by

$$
\sum_{\nu \geq 0} \mathrm{~F}^{\nu} \Gamma\left(\mathrm{fil}_{D^{\prime}} W_{r}\left(\mathscr{K}_{X}\right)\right)
$$

Consider the exact sequence

$$
0 \rightarrow \bigoplus_{\nu \geq 0} \mathrm{fil}_{\lfloor D / p\rfloor} W_{r}\left(\mathscr{K}_{X}\right) \rightarrow \bigoplus_{\nu \geq 0} \mathrm{fil}_{D} W_{r}\left(\mathscr{K}_{X}\right) \rightarrow \mathrm{fil}_{D}^{\mathrm{F}} W_{r}\left(\mathscr{K}_{X}\right) \rightarrow 0
$$

where the third arrow is $\left(w_{v}\right)_{v} \mapsto \sum_{\nu} \mathrm{F}^{\nu} w_{\nu}$, and the second arrow is $\left(w_{v}\right)_{v} \mapsto$ $\left(\mathrm{F} w_{\nu}-w_{\nu-1}\right)_{\nu}$, where we set $w_{-1}=0$. Here $\lfloor D / p\rfloor$ means the largest divisor $E$ such that $p E \leq D$. This yields an exact sequence

$$
\bigoplus_{\nu \geq 0} \Gamma\left(\mathrm{fil}_{D} W_{r}\left(\mathscr{K}_{X}\right)\right) \rightarrow \Gamma\left(\operatorname{fil}_{D}^{\mathrm{F}} W_{r}\left(\mathscr{K}_{X}\right)\right) \rightarrow \bigoplus_{\nu \geq 0} \mathrm{H}^{1}\left(\mathrm{fil}_{\lfloor D / p\rfloor} W_{r}\left(\mathscr{K}_{X}\right)\right) .
$$

$W_{r}\left(\mathscr{K}_{X}\right)$ is the inductive limit of $\operatorname{fil}_{E} W_{r}\left(\mathscr{K}_{X}\right)$, where $E$ ranges over all effective divisors on $X$, and hence

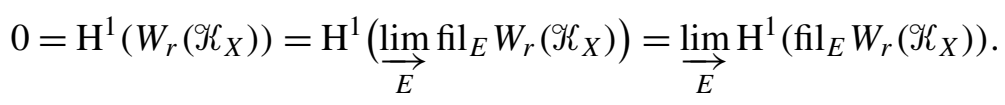


As $\mathrm{H}^{1}\left(\right.$ fil $\left._{\lfloor D / p\rfloor} W_{r}\left(\mathscr{K}_{X}\right)\right)$ is a finite $W_{r}(k)$-module, there is an effective divisor $D^{\prime} \geq D$ such that the image of $\mathrm{H}^{1}\left(\operatorname{fil}_{\lfloor D / p\rfloor} W_{r}\left(\mathscr{K}_{X}\right)\right)$ in $\mathrm{H}^{1}\left(\mathrm{fil}_{\left\lfloor D^{\prime} / p\right\rfloor} W_{r}\left(\mathscr{K}_{X}\right)\right)$ is 0 . Thus the image of $\Gamma\left(\mathrm{fil}_{D}^{\mathrm{F}} W_{r}\left(\mathscr{K}_{X}\right)\right)$ in $\Gamma\left(\mathrm{fil}_{D^{\prime}}^{\mathrm{F}} W_{r}\left(\mathscr{K}_{X}\right)\right)$ is contained in

$$
\sum_{\nu \geq 0} \mathrm{~F}^{\nu} \Gamma\left(\mathrm{fil}_{D^{\prime}} W_{r}\left(\mathscr{K}_{X}\right)\right)
$$

Lemma 3.16. Let $\varphi: X \rightarrow G$ be a rational map from $X$ to a smooth connected algebraic group $G$. Then the following conditions are equivalent:

(i) $\bmod (\varphi) \leq D$.

(ii) $\operatorname{im}\left(\tau_{\varphi}\right) \subset \mathscr{F}_{X, D}$.

Proof. Write $D=\sum_{\mathrm{ht}(q)=1} n_{q} D_{q}$, where $q$ ranges over all points in $X$ of codimension 1 , and $D_{q}$ is the prime divisor associated to $q$. Condition (i) is thus expressed by the condition that for all $q \in X$ of codimension 1 we have

(i) $\bmod _{q}(\varphi) \leq n_{q}$.

Using the canonical splitting of a formal group into an étale and an infinitesimal part, condition (ii) is equivalent to the condition that the following (ii)ét and (ii) inf are satisfied:

(ii)ét $\operatorname{im}\left(\tau_{\varphi, \text { ét }}\right) \subset\left(\mathscr{F}_{X, D}\right)$ ét .

(ii) $)_{\text {inf }} \operatorname{im}\left(\tau_{\varphi, \text { inf }}\right) \subset\left(\mathscr{F}_{X, D}\right)_{\text {inf }}$.

Let $L$ be the affine part of $G$. Remember from Section 2.2.1 that the transformation $\tau_{\varphi}: L^{\vee} \rightarrow \widehat{\operatorname{Div}_{X}}$ is given by $\left\langle\cdot, \ell_{\varphi}\right\rangle$, where $\ell_{\varphi}$ is the image of $\varphi \in G\left(\mathscr{K}_{X}\right)$ in $\Gamma\left(G\left(\mathscr{K}_{X}\right) / G\left(\mathscr{O}_{X}\right)\right) \stackrel{\sim}{\longrightarrow} \Gamma\left(L\left(\mathscr{K}_{X}\right) / L\left(\mathcal{O}_{X}\right)\right)$, and the pairing

$$
\langle\cdot, \cdot\rangle: L^{\vee} \times \Gamma\left(L\left(\mathscr{K}_{X}\right) / L\left(\mathbb{O}_{X}\right)\right) \rightarrow \Gamma\left(\mathbb{G}_{\mathrm{m}}\left(\underline{\mathscr{K}_{X}}\right) / \mathbb{G}_{\mathrm{m}}\left(\underline{\mathbb{O}_{X}}\right)\right) .
$$

is obtained from Cartier duality. Write $L=T \times_{k} U$ as a product of a torus $T$ and a unipotent group $U$. Fix an isomorphism $T \cong\left(\mathbb{G}_{\mathrm{m}}\right)^{m}$ and an isomorphism $U \cong\left(\mathbb{G}_{\mathrm{a}}\right)^{a}$ if $\operatorname{char}(k)=0$, or an embedding $U \subset\left(W_{r}\right)^{a}$ if $\operatorname{char}(k)=p>0$.

Let $\left(t_{j}\right)_{1 \leq j \leq m}$ be the image of $\ell_{\varphi}$ under

$$
\Gamma\left(L\left(\mathscr{K}_{X}\right) / L\left(\mathscr{O}_{X}\right)\right) \rightarrow \Gamma\left(T\left(\mathscr{K}_{X}\right) / T\left(\mathscr{O}_{X}\right)\right) \rightarrow \Gamma\left(\mathbb{G}_{\mathrm{m}}\left(\mathscr{K}_{X}\right) / \mathbb{G}_{\mathrm{m}}\left(\mathscr{O}_{X}\right)\right)^{m}
$$

and $\left(u_{i}\right)_{1 \leq i \leq a}$ be the image of $\ell_{\varphi}$ under

$$
\Gamma\left(L\left(\mathscr{K}_{X}\right) / L\left(\mathbb{O}_{X}\right)\right) \rightarrow \Gamma\left(U\left(\mathscr{K}_{X}\right) / U\left(\mathbb{O}_{X}\right)\right) \rightarrow\left\{\begin{array}{l}
\Gamma\left(\mathbb{G}_{\mathrm{a}}\left(\mathscr{K}_{X}\right) / \mathbb{G}_{\mathrm{a}}\left(\mathbb{O}_{X}\right)\right)^{a}, \\
\Gamma\left(W_{r}\left(\mathscr{K}_{X}\right) / W_{r}\left(\mathbb{O}_{X}\right)\right)^{a}
\end{array}\right.
$$


The étale part of $\tau_{\varphi}$ is

$$
\begin{aligned}
\tau_{\varphi, \text { ét }}: \mathbb{Z}^{m} & \rightarrow \Gamma\left(\mathbb{G}_{\mathrm{m}}\left(\mathscr{K}_{X}\right) / \mathbb{G}_{\mathrm{m}}\left(\mathbb{O}_{X}\right)\right), \\
\left(e_{j}\right)_{1 \leq j \leq m} & \mapsto \prod_{j=1}^{m} t_{j}^{e_{j}} .
\end{aligned}
$$

The image of the infinitesimal part of $\tau_{\varphi}$ is given by the image of

$$
\begin{aligned}
\left.\tilde{\tau}_{\varphi, \text { inf }}: \begin{array}{l}
\left(\widehat{\mathbb{G}}_{\mathrm{a}}\right)^{a} \\
\left.{ }_{r} \widehat{W}\right)^{a}
\end{array}\right\} \rightarrow \Gamma\left(\mathbb{G}_{\mathrm{m}}\left(\mathscr{K}_{X} \otimes \cdot\right) / \mathbb{G}_{\mathrm{m}}\left(\mathbb{O}_{X} \otimes \cdot\right)\right), \\
\left(v_{i}\right)_{1 \leq i \leq a} \mapsto\left\{\begin{array}{l}
\prod_{i=1}^{a} \exp \left(v_{i} u_{i}\right), \\
\prod_{i=1}^{a} \operatorname{Exp}\left(v_{i} \cdot u_{i}, 1\right) ;
\end{array}\right.
\end{aligned}
$$

see Example 1.11 for the pairing $r \widehat{W} \times W_{r} \rightarrow \mathbb{G}_{\mathrm{m}}$. For each $q \in X$ of codimension 1 let $\left(t_{q, i}\right)_{1 \leq j \leq m}$ be a representative in $\mathbb{G}_{\mathrm{m}}\left(\mathscr{K}_{X, q}\right)^{m}$ of the image of $\left(t_{j}\right)_{1 \leq j \leq m}$ under

$$
\Gamma\left(\mathbb{G}_{\mathrm{m}}\left(\mathscr{K}_{X}\right) / \mathbb{G}_{\mathrm{m}}\left(\mathbb{O}_{X}\right)\right)^{m} \rightarrow \mathbb{G}_{\mathrm{m}}\left(\mathscr{K}_{X, q}\right)^{m} / \mathbb{G}_{\mathrm{m}}\left(\mathbb{O}_{X, q}\right)^{m},
$$

and let $\left(u_{q, i}\right)_{1 \leq i \leq a}$ be a representative in $\mathbb{G}_{\mathrm{a}}\left(\mathscr{K}_{X, q}\right)^{a}$ or $W_{r}\left(\mathscr{K}_{X, q}\right)^{a}$ of the image of $\left(u_{i}\right)_{1 \leq i \leq a}$ under

$$
\Gamma\left(\mathbb{G}_{\mathrm{a}}\left(\mathscr{K}_{X}\right) / \mathbb{G}_{\mathrm{a}}\left(\mathscr{O}_{X}\right)\right)^{a} \rightarrow \mathbb{G}_{\mathrm{a}}\left(\mathscr{K}_{X, q}\right)^{a} / \mathbb{G}_{\mathrm{a}}\left(\mathbb{O}_{X, q}\right)^{a}
$$

or

$$
\Gamma\left(W_{r}\left(\mathscr{K}_{X}\right) / W_{r}\left(\mathcal{O}_{X}\right)\right)^{a} \rightarrow W_{r}\left(\mathscr{K}_{X, q}\right)^{a} / W_{r}\left(\mathcal{O}_{X, q}\right)^{a},
$$

respectively. Then (ii)ét is equivalent to the following condition being satisfied for every point $q \in X$ of codimension 1 :

(ii)ét, $q$ If $n_{q}=0$, then $t_{q, j} \in \mathbb{G}_{\mathrm{m}}\left(\mathbb{O}_{X, q}\right)$ for $1 \leq j \leq m$.

On the other hand, condition (ii) $)_{\text {inf }}$ is equivalent to the following condition being satisfied for every point $q \in X$ of codimension 1, according to Definition 3.14 of $\mathscr{F}_{X, D}$ :

(ii) inf, $q$ If $n_{q}=0$, then $u_{q, i} \in \mathbb{G}_{\mathrm{a}}\left(\mathbb{O}_{X, q}\right)$ or $W_{r}\left(\mathbb{O}_{X, q}\right)$ for $1 \leq i \leq a$.

If $n_{q}>0$, then $\mathrm{n}_{q}\left(u_{q, i}\right) \leq n_{q}-1$.

Note that $\varphi \in G\left(\mathscr{O}_{X, q}\right)$ if and only if $t_{q, j} \in \mathbb{G}_{\mathrm{m}}\left(\mathbb{O}_{X, q}\right)$ for $1 \leq j \leq m$ and $u_{q, i} \in \mathbb{G}_{\mathrm{a}}\left(\mathcal{O}_{X, q}\right)$ or $W_{r}\left(\mathcal{O}_{X, q}\right)$ for $1 \leq i \leq a$. By Definition 3.11, for each $q \in X$ of codimension 1

(i) $\bmod _{q}(\varphi) \leq n_{q}$

if and only if (ii)ét, $q$ and (ii) $)_{\text {inf, } q}$ are satisfied.

Now assume $k$ is algebraically closed. Arbitrary perfect base field is considered in Sections 3.2.2 and 3.2.3. 
Theorem 3.17. The category $\operatorname{Mr}(X, D)$ of rational maps of modulus $\leq D$ is equivalent to the category $\operatorname{Mr}_{\mathscr{F}_{X, D}}(X)$ of rational maps that induce a transformation to $\mathscr{F}_{X, D}$.

Proof. According to the definitions of $\operatorname{Mr}(X, D)$ and $\operatorname{Mr}_{\mathscr{F}_{X, D}}(X)$, the statement is due to Lemma 3.16.

Theorem 3.18. The Albanese $\operatorname{Alb}(X, D)$ of $X$ of modulus $D$ exists and is dual (in the sense of 1 -motives) to the 1 -motive $\left[\mathscr{F}_{X, D}^{0, \text { red }} \rightarrow \mathrm{Pic}_{X}^{0, \text { red }}\right]$.

Proof. By Theorem 3.17, $\operatorname{Alb}(X, D)$ is the universal object of $\operatorname{Mr}_{\mathscr{F}_{X, D}}(X)$ (if it exists). A rational map from $X$ to an algebraic group induces a transformation to $\mathscr{F}_{X, D}$ if and only if it induces a transformation to $\mathscr{F}_{X, D}^{0, \text { red }}$, by Lemma 2.6. Since $\mathscr{F}_{X, D}^{0, \text { red }}$ is dual-algebraic (Proposition 3.15), the category $\operatorname{Mr}_{\mathscr{F}_{X, D}}(X)$ admits a universal object (Theorem 2.16), and this universal object is dual to $\left[\mathscr{F}_{X, D}^{0, \text { red }} \rightarrow \operatorname{Pic}_{X}^{0, \text { red }}\right]$ (Remark 2.18).

3.2.2. Descent of the base field. Let $k$ be a perfect field. Let $\bar{k}$ be an algebraic closure of $k$. Let $X$ be a smooth proper variety defined over $k$, and let $D$ be an effective divisor on $X$ rational over $k$.

Theorem 3.19. There exists a k-torsor $\operatorname{Alb}^{(1)}(X, D)$ for an algebraic k-group $\operatorname{Alb}^{(0)}(X, D)$ and rational maps defined over $k$

$$
\operatorname{alb}_{X, D}^{(i)}: X^{2-i} \rightarrow \operatorname{Alb}^{(i)}(X, D)
$$

for $i=1,0$, satisfying the following universal property:

If $\varphi: X \rightarrow G^{(1)}$ is a rational map defined over $k$ to a $k$-torsor $G^{(1)}$ for an algebraic $k$-group $G^{(0)}$, such that $\bmod (\varphi) \leq D$, then there exist a unique homomorphism of $k$-torsors $h^{(1)}: \operatorname{Alb}^{(1)}(X, D) \rightarrow G^{(1)}$ and a unique homomorphism of algebraic $k$-groups $h^{(0)}: \operatorname{Alb}^{(0)}(X, D) \rightarrow G^{(0)}$, defined over $k$, such that $\varphi^{(i)}=h^{(i)} \circ \operatorname{alb}_{X, D}^{(i)}$ for $i=1,0$.

Here $\operatorname{Alb}^{(0)}(X, D)$ is dual to the 1 -motive $\left[\mathscr{F}_{X, D}^{0, \text { red }} \rightarrow \operatorname{Pic}_{X}^{0, \text { red }}\right]$.

Proof. It follows directly from Theorem 2.21 and the definition of the modulus via Galois descent (Definition 3.11).

Corollary 3.20. For every rational map $\varphi: X \rightarrow P$ from $X$ to a torsor $P$ there exists an effective divisor $D$, namely $D=\bmod (\varphi)$, such that $\varphi$ factors through $\operatorname{Alb}^{(1)}(X, D)$.

Proposition 3.21. Let $\mathscr{F}$ be a formal $k$-subgroup of $\underline{\operatorname{Div}}_{X}^{0, \text { red }}$. Then $\mathscr{F}$ is dualalgebraic if and only if there exists an effective divisor $D$, rational over $k$, such that $\mathscr{F}_{\mathcal{F}} \subset \mathscr{F}_{X, D}$. 
Proof. $(\Longrightarrow)$ A formal subgroup of a dual-algebraic group is also dual-algebraic, according to Lemma 1.17 .

$(\Longleftarrow$ ) By Galois descent (possible for formal groups due to Cartier duality) we may assume that $k$ is algebraically closed. Let $D=\bmod \left(\mathrm{alb}_{\mathscr{F}}\right)$ be the modulus of the universal rational map alb $\operatorname{FF}_{\mathscr{F}}: X \rightarrow \operatorname{Alb}_{\mathscr{F}}(X)$ associated to $\mathscr{F} \subset \underline{\operatorname{Div}}_{X}^{0, \text { red }}$. Then by Lemma 3.16, we have $\mathscr{F}_{F}=\operatorname{im}\left(\tau_{\mathrm{alb}_{\mathscr{F}}}\right) \subset \mathscr{F}_{X, D}$.

3.2.3. Functoriality. We specialize the results from Section 2.3 .3 to the case of Albanese varieties with modulus.

Proposition 3.22. Let $\psi: Y \rightarrow X$ be a morphism of smooth proper varieties. Let $D$ be an effective divisor on $X$ intersecting $\psi(Y)$ properly. Then $\psi$ induces a homomorphism of torsors $\mathrm{Alb}^{(1)}{ }_{Y, E}^{X, D}(\psi)$ and a homomorphism of algebraic groups $\operatorname{Alb}_{Y, E}^{(0)} \underset{Y, D}{(\psi),}$

$$
\operatorname{Alb}_{Y, E}^{(i)}(\psi): \operatorname{Alb}^{(i)}(Y, E) \rightarrow \operatorname{Alb}^{(i)}(X, D),
$$

for each effective divisor $E$ on $Y$ satisfying $E \geq\left(D-D_{\text {red }}\right) \cdot Y+(D \cdot Y)_{\text {red }}$, where $B \cdot Y$ denotes the pull-back of a Cartier divisor $B$ on $X$ to $Y$.

Proof. According to Proposition 2.22, for the existence of $\operatorname{Alb}_{Y, E}^{X, D}(\psi)$ it is sufficient to show $\mathscr{F}_{Y, E} \supset \mathscr{F}_{X, D} \cdot Y$. Definition 3.14 of $\mathscr{F}_{X, D}$ implies that this is the case if and only if $\operatorname{Supp}(E) \supset \operatorname{Supp}(D \cdot Y)$ and $E-E_{\text {red }} \geq\left(D-D_{\text {red }}\right) \cdot Y$. But this is equivalent to $E \geq\left(D-D_{\text {red }}\right) \cdot Y+(D \cdot Y)_{\text {red }}$.

Corollary 3.23. If $D$ and $E$ are effective divisors on $X$ with $E \geq D$, then there are canonical surjective homomorphisms $\operatorname{Alb}^{(i)}(X, E) \rightarrow \operatorname{Alb}^{(i)}(X, D)$ for $i=1,0$, given by $\mathrm{Alb}_{X, E}^{(i) X, D}\left(\mathrm{id}_{X}\right)$.

Proof. If $E \geq D$, it is evident that $\operatorname{Alb}^{(i)}(X, E)$ generates $\operatorname{Alb}^{(i)}(X, D)$; thus $\mathrm{Alb}_{X, E}^{(i)} \underset{X, D}{\left(\mathrm{id}_{X}\right)}$ is surjective.

3.3. Jacobian with modulus. Let $C$ be a smooth proper curve over a perfect field $k$, which we assume to be algebraically closed for convenience. Let $D=\sum_{q \in S} n_{q} q$ be an effective divisor on $C$, where $S$ is a finite set of closed points on $C$ and $n_{q}$ are positive integers for $q \in S$. The Jacobian $J(C, D)$ of $C$ of modulus $D$ is by definition the universal object for the category of those morphisms $\varphi$ from $C \backslash S$ to algebraic groups such that $\varphi(\operatorname{div}(f))=0$ for all $f \in \mathscr{K}_{C}$ with $f \equiv 1 \bmod D$. Here we used the definition $\varphi\left(\sum l_{j} c_{j}\right)=\sum l_{j} \varphi\left(c_{j}\right)$ for a divisor $\sum l_{j} c_{j}$ on $C$ with $c_{j} \in C \backslash S$, and " $f \equiv 1 \bmod D$ " means $\mathrm{v}_{q}(1-f) \geq n_{q}$ for all $q \in S$, where $\mathrm{v}_{q}$ is the valuation attached to the point $q \in C$.

Theorem 3.24. The generalized Jacobian $J(C, D)$ of $C$ of modulus $D$ is an extension

$$
0 \rightarrow L(C, D) \rightarrow J(C, D) \rightarrow J(C) \rightarrow 0
$$


of the classical Jacobian $J(C) \cong \mathrm{Pic}_{C}^{0}$ of $C$, which is an abelian variety, by the affine algebraic group $L(C, D)$, which is characterized by

$$
L(C, D)(k)=\frac{\prod_{q \in S} k(q)^{*}}{k^{*}} \times \prod_{q \in S} \frac{1+\mathfrak{m}_{q}}{1+\mathfrak{m}_{q}^{n_{q}}}
$$

where $k(q)$ denotes the residue field and $\mathfrak{m}_{q}$ the maximal ideal at $q \in C$.

Proof. [Serre 1959, V, §3]; see also the summary [ibid., I, no. 1].

Theorem 3.25. The Jacobian with modulus $J(C, D)$ is dual (in the sense of 1motives) to the 1-motive $\left[\mathscr{F}_{C, D}^{0} \rightarrow \mathrm{Pic}_{C}^{0}\right]$, where $\mathscr{F}_{C, D}^{0}=\mathscr{F}_{C, D}^{0, \text { red }}$ is the formal subgroup of $\underline{\mathrm{Div}}_{C}^{0}$ from Definition 3.14, and $\mathscr{F}_{C, D}^{0} \rightarrow \mathrm{Pic}_{C}^{0}$ is the homomorphism induced by the class map $\underline{\operatorname{Div}}_{C}^{0} \rightarrow \underline{\mathrm{Pic}}_{C}^{0}$.

Proof. We have to ensure that the category for which $J(C, D)$ is universal is characterized by the formal group $\mathscr{F}_{C, D}$. The Jacobian $J(C, D)$ of modulus $D$ is by definition the universal object for morphisms $\varphi$ from $C \backslash S$ to algebraic groups satisfying

(i) $\varphi(\operatorname{div}(f))=0$ for all $f \in \mathscr{K}_{C}$ with $f \equiv 1 \bmod D$.

Condition (i) is equivalent to

(ii) $(\varphi, f)_{q}=0$ for all $q \in S$, for all $f \in \mathscr{K}_{C}$ with $f \equiv 1 \bmod D$ at $q$,

where $(\varphi, \cdot) .: \mathscr{K}_{C}^{*} \times C \rightarrow G(k)$ is the local symbol associated to the morphism $\varphi: C \backslash S \rightarrow G$, according to [Serre 1959, I, no. 1, thèoréme 1 and III, §1]. It is shown in [Kato and Russell 2010, Sections 6.1-6.3] that condition (ii) is equivalent to

(iii) $\bmod (\varphi) \leq D$.

Then the assertion is due to Theorems 3.17 and 3.18.

3.4. Relative Chow group with modulus. Let $X$ be a smooth proper variety over an algebraically closed field $k$, and let $D$ be an effective divisor on $X$ and $D_{\text {red }}$ the reduced part of $D$.

Notation 3.26. If $C$ is a curve in $X$, then $v: \widetilde{C} \rightarrow C$ denotes the normalization. For $f \in \mathscr{K}_{C}$, we write $\tilde{f}:=v^{*} f$ for the image of $f$ in $\mathscr{K}_{\widetilde{C}}$. If $\varphi: X \rightarrow G$ is a rational map, we write $\left.\varphi\right|_{\widetilde{C}}:=\left.\varphi\right|_{C} \circ v$ for the composition of $\varphi$ and $v$. If $B$ is a Cartier divisor on $X$ intersecting $C$ properly, then $B \cdot \widetilde{C}$ denotes the pull-back of $B$ to $\widetilde{C}$.

Definition 3.27. Let $\mathrm{Z}_{0}(X \backslash D)$ be the group of 0 -cycles on $X \backslash D$, set

$\mathfrak{R}_{0}(X, D)=\left\{\begin{array}{l|l}(C, f) & \begin{array}{l}C \text { a curve in } X \text { intersecting } \operatorname{Supp}(D) \text { properly, } f \in \mathscr{K}_{C}^{*} \\ \text { such that } \tilde{f} \equiv 1 \bmod \left(D-D_{\text {red }}\right) \cdot \widetilde{C}+(D \cdot \widetilde{C})_{\text {red }}\end{array}\end{array}\right\}$ 
and let $\mathrm{R}_{0}(X, D)$ be the subgroup of $\mathrm{Z}_{0}(X \backslash D)$ generated by the elements $\operatorname{div}(f)_{C}$ with $(C, f) \in \mathfrak{R}_{0}(X, D)$. Then define

$$
\mathrm{CH}_{0}(X, D)=\mathrm{Z}_{0}(X \backslash D) / \mathrm{R}_{0}(X, D) .
$$

Let $\mathrm{CH}_{0}(X, D)^{0}$ be the subgroup of $\mathrm{CH}_{0}(X, D)$ of cycles $\zeta$ with $\left.\operatorname{deg} \zeta\right|_{W}=0$ for all irreducible components $W$ of $X \backslash D$.

Definition 3.28. Let $\mathrm{Mr}^{\mathrm{CH}}(X, D)$ be the category of rational maps from $X$ to algebraic groups defined as follows: The objects of $\mathrm{Mr}^{\mathrm{CH}}(X, D)$ are morphisms $\varphi: X \backslash D \rightarrow G$ whose associated map on 0-cycles of degree zero,

$$
\mathrm{Z}_{0}(X \backslash D)^{0} \rightarrow G(k), \quad \sum l_{i} p_{i} \mapsto \sum l_{i} \varphi\left(p_{i}\right), \quad \text { where } l_{i} \in \mathbb{Z},
$$

factors through a homomorphism of groups $\mathrm{CH}_{0}(X, D)^{0} \rightarrow G(k)$. The morphisms are the ones as in Definition 2.8. We refer to the objects of $\mathrm{Mr}^{\mathrm{CH}}(X, D)$ as rational maps from $X$ to algebraic groups factoring through $\mathrm{CH}_{0}(X, D)^{0}$.

Theorem 3.29. The category $\operatorname{Mr}(X, D)$ of rational maps of modulus $\leq D$ is equivalent to the category $\mathrm{Mr}^{\mathrm{CH}}(X, D)$ of rational maps factoring through $\mathrm{CH}_{0}(X, D)^{0}$. In particular, the Albanese $\operatorname{Alb}(X, D)$ of $X$ of modulus $D$ is the universal quotient of $\mathrm{CH}_{0}(X, D)^{0}$.

Proof. According to the definitions of $\operatorname{Mr}(X, D)$ and $\mathrm{Mr}^{\mathrm{CH}}(X, D)$ the task is to show that for a morphism $\varphi: X \backslash D \rightarrow G$ from $X \backslash D$ to a smooth connected algebraic group $G$ the following conditions are equivalent:

(i) $\bmod (\varphi) \leq D$,

(ii) $\varphi\left(\operatorname{div}(f)_{C}\right)=0$ for all $(C, f) \in \mathfrak{R}_{0}(X, D)$.

Since $\varphi\left(\operatorname{div}(f)_{C}\right)=\left.\varphi\right|_{\widetilde{C}}\left(\operatorname{div}(\tilde{f})_{\widetilde{C}}\right)($ see [Russell 2008, Lemma 3.32]), condition (ii) is equivalent to the condition

(iii) $\bmod \left(\left.\varphi\right|_{\widetilde{C}}\right) \leq\left(D-D_{\text {red }}\right) \cdot \widetilde{C}+(D \cdot \widetilde{C})_{\text {red }}$ for all curves $C$ in $X$ intersecting $\operatorname{Supp}(D)$ properly,

as was seen in the proof of Theorem 3.25 , substituting $D$ by $\left(D-D_{\text {red }}\right) \cdot \widetilde{C}+(D \cdot \widetilde{C})_{\text {red }}$. The equivalence of (i) and (iii) is the content of Lemma 3.30.

Lemma 3.30. Let $\varphi: X \rightarrow G$ be a rational map from $X$ to a smooth connected algebraic group $G$. Then the following conditions are equivalent:

(i) $\bmod (\varphi) \leq D$,

(ii) $\bmod \left(\left.\varphi\right|_{\widetilde{C}}\right) \leq\left(D-D_{\text {red }}\right) \cdot \widetilde{C}+(D \cdot \widetilde{C})_{\text {red }}$ for all curves $C$ in $X$ intersecting $\operatorname{Supp}(D)$ properly. 
Proof. (i) $\Longrightarrow$ (ii) Let $C$ be a curve in $X$ intersecting $D$ properly. As $\varphi$ is regular away from $D$, the restriction $\left.\varphi\right|_{\widetilde{C}}$ of $\varphi$ to $\widetilde{C}$ is regular away from $D \cdot \widetilde{C}$. Hence $\operatorname{Supp}(\bmod (\varphi \mid \widetilde{C})) \subset \operatorname{Supp}(D \cdot \widetilde{C})=\operatorname{Supp}\left(\left(D-D_{\text {red }}\right) \cdot \widetilde{C}+(D \cdot \widetilde{C})_{\text {red }}\right)$. According to Definition 3.11 of the modulus, it is easy to see that $\bmod (\varphi) \leq D=\left(D-D_{\text {red }}\right)+D_{\text {red }}$ implies $\bmod \left(\left.\varphi\right|_{\widetilde{C}}\right) \leq\left(D-D_{\text {red }}\right) \cdot \widetilde{C}+(D \cdot \widetilde{C})_{\text {red }}$.

(ii) $\Longrightarrow$ (i) Let $E:=\bmod (\varphi)$ and $q \in \operatorname{Supp}(E)$ be a point of codimension 1 in $X$. We are going to construct a family of smooth curves $\left\{C_{e}\right\}_{e}$ intersecting $E$ at a fixed point $x \in E_{q}=\overline{\{q\}}$ such that

$$
\lim _{e \rightarrow \infty} \frac{\bmod _{x}\left(\left.\varphi\right|_{C_{e}}\right)}{\mu_{x}\left(\left(E-E_{\mathrm{red}}\right) \cdot C_{e}\right)+1}=1
$$

where $\mu_{x}(E \cdot C)$ denotes the intersection multiplicity of $E$ and $C$ at $x$.

After the construction we will show that the existence of such a family of curves for each $q \in \operatorname{Supp}(E)$ of codimension 1 in $X$ yields the implication (ii) $\Longrightarrow$ (i).

If $\operatorname{char}(k)=0$, it is easy to see that a general curve $C$ in $X$ intersecting $E_{q}$ at a point $x$ satisfies $\bmod _{x}\left(\left.\varphi\right|_{C}\right)=\mu_{x}\left(\left(E-E_{\text {red }}\right) \cdot C\right)+1$. Therefore we suppose that $\operatorname{char}(k)=p>0$. Using the notation of Definition 3.11, let $\left(u_{q, i}\right)_{1 \leq i \leq a} \in W_{r}\left(\mathscr{K}_{X, q}\right)^{a}$ be a representative of the unipotent part of the class of $\varphi \in G\left(\mathscr{K}_{X, q}\right)$ in

$$
G\left(\mathscr{K}_{X, q}\right) / G\left(\mathcal{O}_{X, q}\right)=L\left(\mathscr{K}_{X, q}\right) / L\left(\mathcal{O}_{X, q}\right) .
$$

Then $\bmod _{q}(\varphi)=1+\mathrm{n}_{q}\left(u_{q, i}\right)$ for some $1 \leq i \leq a$. Set $n:=\mathrm{n}_{q}\left(u_{q, i}\right)$. Let $t \in \mathfrak{m}_{X, q}$ be a uniformizer at $q$. Let

$$
\sum_{\nu} \mathrm{F}^{v} \otimes \omega_{\nu} \otimes t^{-n} \in k[\mathrm{~F}] \otimes_{k} \Omega_{X, q}(\log q) \otimes_{\mathcal{O}_{X, q}} \mathfrak{m}_{X, q}^{-n}
$$

be a representative of $\overline{\mathfrak{d}}_{n q}\left(u_{q, i}\right) \in \overline{\mathfrak{D}}_{n q}$ (Proposition 3.7). Choose a regular closed point $x \in E_{q}$ such that $t$ is a local equation for $E_{q}$ at $x$ and $\omega_{\nu}$ is regular and nonzero at $x$ for some $v$. We may assume that $\operatorname{dim} X=2$ via cutting down by hyperplanes through $x$ transversal to $E_{q}$. Let $s \in \mathfrak{m}_{X, x}$ be a local parameter at $x$ that gives a uniformizer of $O_{E_{q}, x}$. Define a curve $C_{e}$ locally around $x$ by the equation $t=s^{e}$ for $e \geq 1$. Note that $E-E_{\text {red }}$ is locally defined by the equation $t^{n}=0$. Then

$$
\mu_{x}\left(\left(E-E_{\mathrm{red}}\right) \cdot C_{e}\right)=\operatorname{dim}_{k} \frac{\mathrm{O}_{X, x}}{\left(t^{n}, t-s^{e}\right)}=n e .
$$

We can write $\omega_{\nu}=g \mathrm{~d} s+h \mathrm{~d} \log t$ with $g, h \in \mathbb{O}_{X, q}$ and the values at $x$ are $g(x) \neq 0$ if $\overline{\mathfrak{d}}_{n q}\left(u_{q, i}\right) \in b \overline{\mathfrak{D}}_{n q}$, and $h(x) \neq 0$ if $\overline{\mathfrak{d}}_{n q}\left(u_{q, i}\right) \in \overline{\mathfrak{D}}_{n q} \backslash^{b} \overline{\mathfrak{D}}_{n q}$ and $x$ in general position (what we assume), for some $v$. The restriction of $t^{-n} \omega_{v}$ to $C_{e}$ is

$$
\left.t^{-n} \omega_{\nu}\right|_{C_{e}}=s^{-n e} g \mathrm{~d} s+s^{-n e} h \mathrm{~d} \log s^{e}=s^{1-n e} g \mathrm{~d} \log s+e s^{-n e} h \mathrm{~d} \log s,
$$


and the class of $\left.t^{-n} \omega_{\nu}\right|_{C_{e}}$ is nonzero in

$$
\begin{cases}\Omega_{C_{e}, x}(\log x) \otimes_{\mathcal{O}_{e}, x} \mathfrak{m}_{C_{e}, x}^{-n e} / \mathfrak{m}_{C_{e}, x}^{1-n e} & \text { if } \overline{\mathfrak{d}}_{n q}\left(u_{q, i}\right) \in \overline{\mathfrak{D}}_{n q} \backslash \mathrm{b} \overline{\mathfrak{D}}_{n q} \text { and } p \nmid e, \\ \Omega_{C_{e}, x}(\log x) \otimes_{\mathcal{O}_{e, x}} \mathfrak{m}_{C_{e}, x}^{1-n e} / \mathfrak{m}_{C_{e}, x}^{2-n e} & \text { if } \overline{\mathfrak{d}}_{n q}\left(u_{q, i}\right) \in{ }^{b} \overline{\mathfrak{D}}_{n q} .\end{cases}
$$

Lemma 3.10 assures that the modulus of $\left.\varphi\right|_{C_{e}}$ is computed from the restriction (of a representative) of $\overline{\mathfrak{d}}_{n q}\left(u_{q, i}\right)$ to $C_{e}$, for $e$ large enough such that $n e-1>\lfloor n e / p\rfloor$ (this is satisfied for $e>2$ ). Thus we have

$$
\begin{aligned}
\mathrm{n}_{x}\left(\left.u_{q, i}\right|_{C_{e}}\right) & = \begin{cases}n e & \text { if } \overline{\mathfrak{d}}_{n q}\left(u_{q, i}\right) \in \overline{\mathfrak{D}}_{n q} \backslash \mathrm{b} \overline{\mathfrak{D}}_{n q} \text { and } p \nmid e, \\
n e-1 & \text { if } \overline{\mathfrak{d}}_{n q}\left(u_{q, i}\right) \in{ }^{b} \overline{\mathfrak{D}}_{n q},\end{cases} \\
\bmod _{x}\left(\left.\varphi\right|_{C_{e}}\right) & = \begin{cases}n e+1 & \text { if } \overline{\mathfrak{d}}_{n q}\left(u_{q, i}\right) \in \overline{\mathfrak{D}}_{n q} \backslash \mathrm{b} \overline{\mathfrak{D}}_{n q} \text { and } p \nmid e, \\
n e & \text { if } \overline{\mathfrak{d}}_{n q}\left(u_{q, i}\right) \in{ }^{b} \overline{\mathfrak{D}}_{n q} .\end{cases}
\end{aligned}
$$

Then

$$
\lim _{\substack{e \rightarrow \infty \\ p \nmid e}} \frac{\bmod _{x}\left(\left.\varphi\right|_{C_{e}}\right)}{\mu_{x}\left(\left(E-E_{\mathrm{red}}\right) \cdot C_{e}\right)+1}=1 .
$$

Now we show that "not (i) implies not (ii)". Suppose $E:=\bmod (\varphi) \not \subset D$. Then there is a point $q \in \operatorname{Supp}(E)$ of codimension 1 in $X$ such that $\mu_{q}(E)>\mu_{q}(D)$, where $\mu_{q}$ is the multiplicity at $q$. By the construction above there is a sequence of curves $\left\{C_{e}\right\}_{e}$ in $X$ intersecting $E$ at a fixed point $x \in E_{q}$ such that

$$
\lim _{\substack{e \rightarrow \infty \\ p \nmid e}} \frac{\bmod _{x}\left(\left.\varphi\right|_{C_{e}}\right)}{\mu_{x}\left(\left(E-E_{\mathrm{red}}\right) \cdot C_{e}\right)+1}=1 .
$$

If $\mu_{q}(D) \neq 0$, then since

$$
\sup _{e \geq 0} \frac{\mu_{x}\left(\left(D-D_{\mathrm{red}}\right) \cdot C_{e}\right)+1}{\mu_{x}\left(\left(E-E_{\mathrm{red}}\right) \cdot C_{e}\right)+1}<1,
$$

there is $e$ such that $\bmod _{x}\left(\left.\varphi\right|_{C_{e}}\right)>\mu_{x}\left(\left(D-D_{\text {red }}\right) \cdot C_{e}\right)+1$. If $\mu_{q}(D)=0$, then

$$
0 \neq \bmod \left(\left.\varphi\right|_{C_{e}}\right)_{x}>\mu_{x}\left(\left(D-D_{\mathrm{red}}\right) \cdot C_{e}+\left(D \cdot C_{e}\right)_{\mathrm{red}}\right)=0 .
$$

Thus $\bmod \left(\left.\varphi\right|_{C_{e}}\right) \not \subset\left(D-D_{\mathrm{red}}\right) \cdot C_{e}+\left(D \cdot C_{e}\right)_{\mathrm{red}}$.

\section{Acknowledgement}

I owe many thanks to Kazuya Kato for his hospitality, help and support. His influence on this work is considerable. I also thank the referee for helpful suggestions. In particular I replaced my original proof of Theorem 1.19 by a shorter argument due to the referee. 


\section{References}

[Barbieri-Viale and Bertapelle 2009] L. Barbieri-Viale and A. Bertapelle, "Sharp de Rham realization”, Adv. Math. 222:4 (2009), 1308-1338. MR 2010i:14002 Zbl 1216.14006

[Bosch et al. 1990] S. Bosch, W. Lütkebohmert, and M. Raynaud, Néron models, Ergebnisse der Mathematik und ihrer Grenzgebiete (3) 21, Springer, Berlin, 1990. MR 91i:14034 Zbl 0705.14001

[Brylinski 1983] J.-L. Brylinski, "Théorie du corps de classes de Kato et revêtements abéliens de surfaces”, Ann. Inst. Fourier (Grenoble) 33:3 (1983), 23-38. MR 85f:11088 Zbl 0524.12008

[Deligne 1971] P. Deligne, “Théorie de Hodge, II”, Inst. Hautes Études Sci. Publ. Math. 40 (1971), 5-57. MR 58 \#16653a Zbl 0219.14007

[Demazure 1972] M. Demazure, Lectures on p-divisible groups, Lecture Notes in Mathematics 302, Springer, Berlin, 1972. MR 88a:14049 Zbl 0247.14010

[Demazure and Gabriel 1970] M. Demazure and P. Gabriel, Groupes algébriques, I: Géométrie algébrique, généralités, groupes commutatifs, Masson \& Cie, Paris, 1970. MR 46 \#1800

[Fontaine 1977] J.-M. Fontaine, Groupes p-divisibles sur les corps locaux (Orsay, 1992), Astérisque 47-48, Société Mathématique de France, Paris, 1977. MR 58 \#16699 Zbl 0377.14009

[Kato and Russell 2010] K. Kato and H. Russell, "Modulus of a rational map into a commutative algebraic group”, Kyoto J. Math. 50:3 (2010), 607-622. MR 2011j:14098 Zbl 1206.14069

[Kato and Russell 2012] K. Kato and H. Russell, "Albanese varieties with modulus and Hodge theory", Ann. Inst. Fourier 62:2 (2012), 783-806.

[Kato and Saito 1983] K. Kato and S. Saito, "Two-dimensional class field theory", pp. 103-152 in Galois groups and their representations (Nagoya, 1981), edited by Y. Ihara, Adv. Stud. Pure Math. 2 , North-Holland, Amsterdam, 1983. MR 87a:11060 Zbl 0544.12011

[Lang 1959] S. Lang, Abelian varieties, Interscience Tracts in Pure and Applied Mathematics 7 , Interscience Publishers, New York, 1959. MR 21 \#4959 Zbl 0099.16103

[Laumon 1996] G. Laumon, “Transformation de Fourier généralisée”, preprint, 1996. arXiv alggeom/9603004v1

[Matsusaka 1952] T. Matsusaka, "On the algebraic construction of the Picard variety, II", Jap. J. Math. 22 (1952), 51-62. MR 15,983b Zbl 0049.22801

[Milne 1980] J. S. Milne, Étale cohomology, Princeton Mathematical Series 33, Princeton University Press, 1980. MR 81j:14002 Zbl 0433.14012

[Önsiper 1989] H. Önsiper, "Generalized Albanese varieties for surfaces in characteristic $p>0$ ", Duke Math. J. 59:2 (1989), 359-364. MR 90h:14025 Zbl 0753.14039

[Oort 1966] F. Oort, Commutative group schemes, Lecture Notes in Mathematics 15, Springer, Berlin, 1966. MR 35 \#4229 Zbl 0216.05603

[Russell 2008] H. Russell, “Generalized Albanese and its dual”, J. Math. Kyoto Univ. 48:4 (2008), 907-949. MR 2010b:14008 Zbl 1170.14005

[Serre 1958-1959] J.-P. Serre, "Morphismes universels et variétés d'Albanese”, pp. 1-22, Exp. No. 10 in Variétés de Picard, Séminaire C. Chevalley 4, Secrétariat mathématique, Paris, 1958-1959. Zbl 0123.13903

[Serre 1959] J.-P. Serre, Groupes algébriques et corps de classes, Publications de l'institut de mathématique de l'université 7, Hermann, Paris, 1959. MR 21 \#1973 Zbl 0097.35604

[SGA3 1970] M. Demazure and A. Grothendieck (editors), Schémas en groupes, I: Propriétés générales des schémas en groupes (SGA3), Lecture Notes in Mathematics 151, Springer, Berlin, 1970. MR 43 \#223a Zbl 0207.51401 
[Waterhouse 1979] W. C. Waterhouse, Introduction to affine group schemes, Graduate Texts in Mathematics 66, Springer, New York, 1979. MR 82e:14003 Zbl 0442.14017

Communicated by Brian Conrad

Received 2011-02-18 Revised 2012-04-07 Accepted 2012-05-17

henrik.russell@math.fu-berlin.de

Freie Universität Berlin, Mathematik und Informatik, Arnimallee 3, 14195 Berlin, Germany 


\section{Algebra \& Number Theory}

msp.org/ant

\section{EDITORS}

MANAGING EDITOR

Bjorn Poonen

Massachusetts Institute of Technology

Cambridge, USA

\author{
EDITORIAL BOARD CHAIR \\ David Eisenbud \\ University of California \\ Berkeley, USA
}

\section{BOARD OF EDITORS}

Georgia Benkart

Dave Benson

Richard E. Borcherds

John H. Coates

J-L. Colliot-Thélène

Brian D. Conrad

Hélène Esnault

Hubert Flenner

Edward Frenkel

Andrew Granville

Joseph Gubeladze

Ehud Hrushovski

Craig Huneke

Mikhail Kapranov

Yujiro Kawamata

János Kollár

Yuri Manin

Barry Mazur

Philippe Michel
University of Wisconsin, Madison, USA

University of Aberdeen, Scotland

University of California, Berkeley, USA

University of Cambridge, UK

CNRS, Université Paris-Sud, France

University of Michigan, USA

Freie Universität Berlin, Germany

Ruhr-Universität, Germany

University of California, Berkeley, USA

Université de Montréal, Canada

San Francisco State University, USA

Hebrew University, Israel

University of Virginia, USA

Yale University, USA

University of Tokyo, Japan

Princeton University, USA

Northwestern University, USA

Harvard University, USA

École Polytechnique Fédérale de Lausanne
Susan Montgomery

Shigefumi Mori

Raman Parimala

Jonathan Pila

Victor Reiner

Karl Rubin

Peter Sarnak

Joseph H. Silverman

Michael Singer

Vasudevan Srinivas

J. Toby Stafford

Bernd Sturmfels

Richard Taylor

Ravi Vakil

Michel van den Bergh

Marie-France Vignéras

Kei-Ichi Watanabe

Efim Zelmanov
University of Southern California, USA

RIMS, Kyoto University, Japan

Emory University, USA

University of Oxford, UK

University of Minnesota, USA

University of California, Irvine, USA

Princeton University, USA

Brown University, USA

North Carolina State University, USA

Tata Inst. of Fund. Research, India

University of Michigan, USA

University of California, Berkeley, USA

Harvard University, USA

Stanford University, USA

Hasselt University, Belgium

Université Paris VII, France

Nihon University, Japan

University of California, San Diego, USA

\section{PRODUCTION}

production@msp.org

Silvio Levy, Scientific Editor

See inside back cover or msp.org/ant for submission instructions.

The subscription price for 2013 is US \$200/year for the electronic version, and \$350/year ( $\$ 40$, if shipping outside the US) for print and electronic. Subscriptions, requests for back issues and changes of subscribers address should be sent to MSP.

Algebra \& Number Theory (ISSN 1944-7833 electronic, 1937-0652 printed) at Mathematical Sciences Publishers, 798 Evans Hall \#3840, c/o University of California, Berkeley, CA 94720-3840 is published continuously online. Periodical rate postage paid at Berkeley, CA 94704, and additional mailing offices.

ANT peer review and production are managed by EditFLOW ${ }^{\circledR}$ from Mathematical Sciences Publishers.

\section{PUBLISHED BY}

- mathematical sciences publishers

nonprofit scientific publishing

http://msp.org/

(C) 2013 Mathematical Sciences Publishers 


\section{Algebra \& Number Theory}

$\begin{array}{lll}\text { Volume } 7 & \text { No. } 4 \quad 2013\end{array}$

Explicit Chabauty over number fields

SAMIR SIKSEK

Moduli spaces for point modules on naïve blowups

Thomas A. Nevins and Susan J. Sierra

Density of rational points on certain surfaces

Sir Peter SWInNerton-Dyer

HENRIK RUSSELL

Chai's conjecture and Fubini properties of dimensional motivic integration

RAF Cluckers, François LoESER and JohanNes NiCAISE

SHUNSUKE TAKAGI

Finitely presented exponential fields

JONATHAN KIRBY

On a problem of Arnold: The average multiplicative order of a given integer

PÄr Kurlberg and CARL POMERANCE

An analogue of Sturm's theorem for Hilbert modular forms 